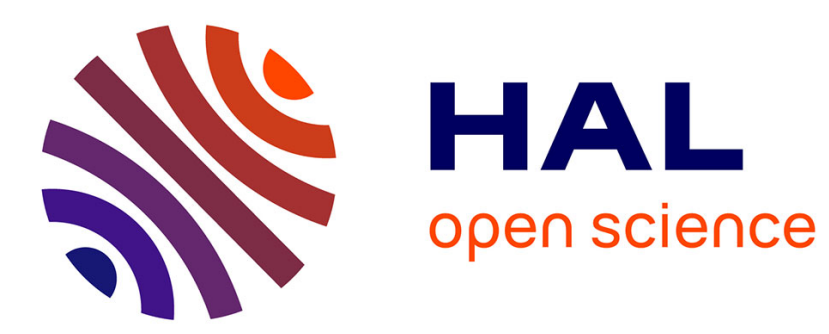

\title{
Towards an Americanization of French Metropolitan Areas? \\ Vincent Hoffmann-Martinot
}

\section{To cite this version:}

Vincent Hoffmann-Martinot. Towards an Americanization of French Metropolitan Areas?. 2004. halshs-00006541

\section{HAL Id: halshs-00006541 https://shs.hal.science/halshs-00006541}

Preprint submitted on 1 Dec 2005

HAL is a multi-disciplinary open access archive for the deposit and dissemination of scientific research documents, whether they are published or not. The documents may come from teaching and research institutions in France or abroad, or from public or private research centers.
L'archive ouverte pluridisciplinaire HAL, est destinée au dépôt et à la diffusion de documents scientifiques de niveau recherche, publiés ou non, émanant des établissements d'enseignement et de recherche français ou étrangers, des laboratoires publics ou privés. 


\title{
TOWARDS AN AMERICANIZATION OF FRENCH METROPOLITAN AREAS ?
}

\author{
Vincent Hoffmann-Martinot \\ Directeur de Recherche au CNRS \\ CERVL-CNRS/ IEP de Bordeaux \\ Domaine universitaire \\ 11, allée Ausone \\ 33607 Pessac Cedex/ France \\ e-mail : v.hoffmann-martinot@wanadoo.fr
}

Paper presented at the Conference of the International Metropolitan Observatory, 9-10 January 2004, Bordeaux, Pôle Universitaire de Bordeaux.

The author expresses his gratitude to the LASMAS-IdL and to Alexandre Kych for having allowed him to use 1999 census data in application of the exchange data agreement between CNRS and INSEE, but also to Monique Perronnet-Menault (TEMIBER-CNRS, Bordeaux) for her precious help in producing maps of French urban areas. 


\section{Metropolization and urban sprawl in French urban areas}

There are two main official measures of urbanization defined by the French census, INSEE (Julien 2000):

1. the urban unit (unite urbaine) fits the agglomeration concept. It includes two categories :

a. the urban agglomeration: a group of communes on the territory of which lives a population of at least 2.000 inhabitants + a continuity of the built environment, i.e. there are no gaps (agricultural land, forest) of more than 200 metres (a criterium also used in Switzerland)

b. the isolated city : the same definition applied to a sole commune

France had 1.995 urban units at the last census conduted in 1999.

2. officially introduced in 1996 in order to measure in a better way the so-called périurbanisation phenomenon (urban sprawl towards distant suburbs), the metropolitan area (aire urbaine) includes :

a. an urban pole (pôle urbain) = an urban unit with at least 5.000 jobs, where less than $40 \%$ of the working population works outside

b. an exurban ring (couronne périurbaine), composed of rural communes or urban units whose working population works in the rest of the urban area

The metropolitan area (see Appendix 1) was selected as unit of analysis in order to encompass the core city or cities, suburbs, and exurbs, the latter being often not contiguous to other communes. As in other countries like Sweden, the urban area is defined according to the municipal economic and employment interactions rather than to the continuity of the built environment.

According to the last census conducted in 1999, there are 354 urban areas concentrating $77 \%$ of the population (45 millions), compared with $73 \%$ in 1990. En 1999, 3,8 millions d'habitants supplémentaires ont été dénombrés dans les aires urbaines. Cet accroissement est la conjonction de deux phénomènes. D'une part, la population a augmenté 
de 1,5 million dans les limites de 1990 des aires urbaines. D'autre part, 2,3 millions d'habitants ont été gagnés par l'extension géographique des aires urbaines entre 1990 et 1999.

Même si la notion d'aire urbaine n'a été appliquée qu'à partir du dernier recensement de 1999, il est possible de reconstituer les aires urbaines depuis 1968 par rétropolation, en s'appuyant notamment sur les travaux de Julien (2003). Il apparaît alors que le nombre d'aires urbaines est resté relativement stable : 319 en 1968, 347 en 1975, 359 en 1982, 361 en 1990, et 354 en 1999. Au niveau global, la figure 1 montre que l'étalement urbain a certes affecté les pôles urbains ou agglomérations au sens traditionnel, le nombre de communes qu'ils englobent augmentant de 48\% en trois décennies (de 2.098 à 3 .100). Mais l'essentiel de cette évolution a touché la ceinture des banlieues plus éloignées, appelées en France communes périurbaines, dont le nombre de communes est multiplié sur la même période par $651 \%$ (de 1.440 à 10.808). Alors que la ville est traditionnellement définie par la contiguité de l'habitat, on appelle périurbaine une ceinture située en dehors de la ville et prenant la forme d'un espace mixte où se trouvent à la fois des ménages occupant des emplois urbains et des agriculteurs. Le périurbain est donc un espace rural au sens où l'essentiel des sols y sont dédiés à des activités productives agricoles ; mais c'est aussi un espace urbain au sens où la majorité de la population active qui y habite travaille dans une ville, en effectuant des migrations alternantes ((sur le développement des communes périurbaines: Cavailhès et al. 2002, Péguy 2000). 
Figure 1. Measuring urban sprawl in all French urban areas: evolution of the number of communes in urban poles and urban belts from 1968 to 1999

1968

1999

1975

10808

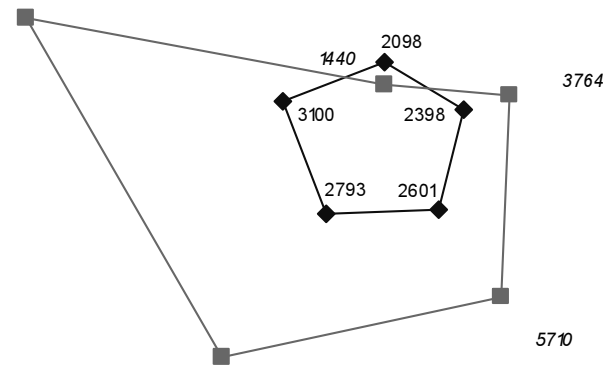

7892

1990

1982 
Table 1. The 42 French

metropolitan areas over 200.000

inhabitants (1999)

\begin{tabular}{lr}
\hline AIRE URBAINE 1999 & $\begin{array}{r}\text { Population } \\
\text { totale }\end{array}$ \\
\hline PARIS & 11173886 \\
LYON & 1647722 \\
MARSEILLE-AIX-EN-PRO. & 1516086 \\
LILLE & 1142887 \\
TOULOUSE & 964914 \\
NICE & 933551 \\
BORDEAUX & 925429 \\
NANTES & 711241 \\
STRASBOURG & 611971 \\
TOULON & 564740 \\
DOUAI-LENS & 552635 \\
RENNES & 521183 \\
ROUEN & 518340 \\
GRENOBLE & 514586 \\
MONTPELLIER & 459946 \\
METZ & 429544 \\
NANCY & 410405 \\
CLERMONT-FERRAND & 409533 \\
VALENCIENNES & 399581 \\
TOURS & 376131 \\
CAEN & 370752 \\
ORLEANS & 355770 \\
ANGERS & 332737 \\
DIJON & 326886 \\
SAINT-ETIENNE & 321953 \\
BREST & 303528 \\
LE HAVRE & 296795 \\
LE MANS & 293094 \\
REIMS & 291701 \\
AVIGNON & 290524 \\
AMIENS & 270809 \\
MULHOUSE & 270752 \\
BETHUNE & 268435 \\
DUNKERQUE & 265906 \\
PERPIGNAN & 249041 \\
LIMOGES & 247881 \\
BESANCON & 222388 \\
NIMES & 221380 \\
PAU & 216868 \\
BAYONNE & 214039 \\
GENEVE-ANNEMASSE & 212451 \\
POITIERS & 209250 \\
MEDIANE & 363261 \\
\hline N $=6785$ & \\
SOUrCe : INSEE & \\
\hline
\end{tabular}


Si les actifs travaillent effectivement dans l'agglomération dense, ils habitent de plus en plus dans des quartiers excentrés qui se densifient alors petit à petit. Cette tendance illustre aussi parfois la perte de dynamisme des communes périphériques: incapables d'offrir des emplois en remplacement des emplois traditionnels (agriculteurs, artisans), ces petites communes deviennent de plus en plus dépendantes économiquement du pôle à proximité, obligeant leur population à une mobilité accrue.

42 metropolitan areas have a population greater than 200.000 inhabitants. These biggest metropolitan areas are represented in Table 1 and Appendix 2. Maps of the metropolitan areas of Paris and Bordeaux are in Appendix 3 and Appendix 4.

Le recensement de 1999 illustre deux phénomènes : la polarisation toujours plus forte autour de grandes métropoles et la confirmation de l'étalement urbain (Hoffmann-Martinot 2000). Aujourd'hui, la moitié de la croissance démographique entre 1990 et 1999 est assurée par 11 aires urbaines, quand il en fallait 19 au début des années 80 .

L'accroissement démographique se fait donc autour de quelques grandes villes, avec toujours en tête Paris, Lyon et Toulouse. Cependant, la progression démographique de Paris ralentit ( $+0,32 \%$ annuel) et se situe en dessous de la moyenne nationale. A l'inverse, plusieurs métropoles ont connu un dynamisme extrêmement fort avec un accroissement de leur population au moins deux fois plus important que dans le reste du pays: Montpellier d'abord ( $+1,88 \%$ annuel), Toulouse, Rennes et Nantes par exemple. Globalement, c'est dans le grand Sud-Est et dans le Val de Loire que le dynamisme est le plus fort.

Alors que l'aire urbaine de Paris ne couvrait en 1968 que 22\% de l'Ile-de-France, elle est aujourd'hui un vaste territoire de $14.518 \mathrm{~km}^{2}$ qui dépasse de $21 \%$ la superficie de l'Ile-deFrance, décalé vers le nord-ouest par rapport aux limites administratives de la région Ile-deFrance. Elle couvre entièrement sept des huit départements franciliens (Essonne, Hauts-deSeine, Paris, Seine-Saint-Denis, Val-de-Marne, Val-d'Oise, Yvelines), 79\% du territoire de la Seine-et-Marne, et déborde des frontières régionales, dans six départements limitrophes. 99\% de la population francilienne vit dans l'aire urbaine de Paris. 
Composée aujourd'hui de 1.584 communes (Appendix 3), dont près de 400 hors Ilede-France, l'aire urbaine de Paris s'est agrandie, entre 1990 et 1999, de 429 communes. Dans sa partie francilienne, elle a progressé sur les deux départements qu'elle ne couvrait pas entièrement, l'Essonne et la Seine-et-Marne. En 1999, seulement 89 communes franciliennes sur 1.281, toutes au sud-est de la Seine-et-Marne, échappent à l'aire urbaine de Paris. Cette extension vers l'est a débordé sur trois départements voisins, raccrochant à la couronne périurbaine parisienne 23 communes de l'Aisne, 3 de la Marne et 10 du Loiret. Mais c'est au nord et à l'ouest de la région, dans l'Oise, l'Eure et l'Eure-et-Loir que l'attraction de l'agglomération parisienne et de sa couronne est la plus forte. Entre 1990 et 1999, 135 nouvelles communes se sont raccrochées à l'aire urbaine de Paris dans ces trois départements : l'Oise a cédé 52 communes supplémentaires, l'Eure 45, et l'Eure-et-Loir 38. Globalement, ces communes étaient en 1990, à parts égales, soit multipolarisées soit dans un espace à dominante rurale.

Particulièrement spectaculaire dans l'aire urbaine de Paris, l'étalement urbain est également manifeste dans les autres grandes métropoles. De 90 communes en 1968, celle de Toulouse s'est progressivement étendue à 152 en 1975, 195 en 1982, 255 en 1990, et 342 en 1999. Elle couvre désormais la majeure partie du département de la Haute-Garonne (308 des 588 communes) et déborde sur les départements de l'Ariège (6 communes), l'Aude (2 communes), le Gers (7 communes), le Tarn (14 communes) et le Tarn-et-Garonne (5 communes).

On observe la même tendance extensive dans nos autres aires urbaines de plus de 200.000 habitants : le nombre de communes s'élève ainsi sensiblement à Besançon (de 186 à 234), Bordeaux (149 à 191), Clermont-Ferrand (99 à 147), Limoges (53 à 78), Pau (91 à 142), Poitiers (56 à 83), Rouen (142 à 189), Strasbourg (128 à 182).

Cette évolution souligne cruellement le caractère obsolète du découpage territorial en collectivités territoriales créées il y a deux siècles comme les départements. Ceux-ci sont notamment chargés d'aider au développement de la coopération et de la solidarité intercommunales à l'intérieur de leurs territoires respectifs, notamment dans les secteurs des transports collectifs, de l'aide sociale et de l'éducation. Or la configuration actuelle des aires urbaines comme nouveaux espaces de travail et de consommation déborde largement le cadre départemental classique pour la majorité d'entre elles (outre Paris et Toulouse, on peut citer 
comme exemple l'aire urbaine de Lyon qui s'étend à la fois sur l'Ain, l'Isère, la Loire et le Rhône), voire régional (aires urbaines d'Avignon, de Paris ou de Reims), ou même national (aire urbaine de Genève-Annemasse ${ }^{1}$ ).

Un même constat d'obsolescence ou d'inadaptation institutionnelle s'applique aux organismes inter- ou supra-communaux qui continuent à gérer une partie devenue fort réduite des aires urbaines en extension. Mise en place en 1968, la Communauté Urbaine de Bordeaux demeure ainsi compétente pour ce qui constituait l'essentiel de l'aire urbaine de l'époque ou du moins de son pôle urbain, soit 27 communes aujourd'hui noyées dans un espace d'interactions urbaines beaucoup plus vaste.

\section{Un niveau élevé et variable de fragmentation géo-politique}

Le territoire français est particulièrement fragmenté : le nombre de communes est de loin le plus élevé du continent européen puisqu'il s'élève selon le dernier recensement à 36.565 pour la France métropolitaine. Il correspond donc approximativement au nombre de municipalités (19.429) et de townships (16.504) des Etats-Unis (U.S. Census Bureau 2002). La plupart des communes françaises sont peu peuplées : 76\% des communes ont moins de 1.000 habitants regroupant seulement un Français sur six. Dans 3.011 communes, la population est inférieure à 500 habitants, et on compte même des communes sans population, en particulier, dans le département de la Meuse, les six communes-martyres «mortes pour la France » à la suite des terribles combats de la région de Verdun au cours de la première guerre mondiale (Beaumont-en-Verdunois, Bezonvaux, Cumières-le-Mort-Homme, Fleury-devantDouaumont, Haumont-près- Samogneux, et Louvement-Côte-du-Poivre).

Parmi les raisons expliquant ce niveau général particulièrement élevé de fragmentation territoriale, l'une a été brillamment exposée en 1924 devant l'Académie de Prusse par Hintze (1962). Selon lui, la constitution des Etats européens entre le Moyen-Age et le XIXème siècle a suivi deux voies distinctes selon la structure de la féodalité qu'ils ont ou non expérimentée. Dans les pays où la féodalité fut absente, faiblement ou tardivement présente, ou bien où elle ne s'accompagnait pas d'une transmission héréditaire des bénéfices (aboutissant à la fragmentation des biens) - l'Angleterre, le Danemark, la Norvège, la Suède, la Pologne, la

\footnotetext{
${ }^{1}$ Dans les analyses de cette étude, seule la partie française de cette région urbaine frontalière est cependant prise en considération.
} 
Hongrie, la Bohême, l'Italie du Sud, et la Castille - les anciens grands territoires ruraux se sont maintenus et ont donné naissance à des collectivités territoriales supra-communales (höhere Kommunalverbände tels que les counties anglais, les vö̈vodies polonaises ou les comitats hongrois), éclipsant en grande partie les unités de base, les municipalités. Par contre, les pays qui formaient l'ancien empire carolingien - la France, l'Allemagne à l'Ouest de l'Elbe, l'Italie du Nord et du Centre, l'Aragon - ont connu entre le Xème et le XIIème siècle un scénario inverse: la logique des successions héréditaires inscrite dans le développement de la féodalité a entraîné un éclatement, une véritable «pulvérisation $»^{2}$ des vastes entités territoriales traditionnelles (comtés, provinces) et un redécoupage complet opéré par les autorités seigneuriales et monarchiques basé sur de petites collectivités.

Mais, à partir du XIXème siècle, alors que plusieurs vagues de fusions vont contribuer à réduire sensiblement le nombre de communes allemandes - le dernier grand mouvement des années 1960-70 qui divise par trois leur nombre en Allemagne de l'Ouest (de 25.000 à 8.500) ne fait que prolonger une tendance de fond amorcée dès les années 1880 - les limites des communes françaises demeurent quasiment inchangées en France depuis des siècles, ce qui distingue nettement les villes françaises des cités allemandes et nord-européennes qui ont englobé progressivement une partie de leurs périphéries (Hoffmann-Martinot 2002). Même un pays par tradition fortement attaché au maintien de ses nombreuses petites communes comme la Suisse a poursuivi depuis les années 1990, notamment dans onze de ses cantons, une politique de regroupement des municipalités (Kübler et Ladner 2003, Ladner et Steiner 2003).

La grande majorité des Français vivent donc aujourd'hui dans des aires urbaines, la moitié d'entre eux dans celles de plus de 200.000 habitants et plus d'un sixième (11.173.886) dans la seule aire urbaine de Paris. La fragmentation générale du territoire se reflète également à leur échelle (Tableau 2).

\footnotetext{
${ }^{2}$ Hintze, op. cit. : 227
} 
Table 2. The geopolitical fragmentation of the 42 French metropolitan areas over 200.000 inhabitants (1999)

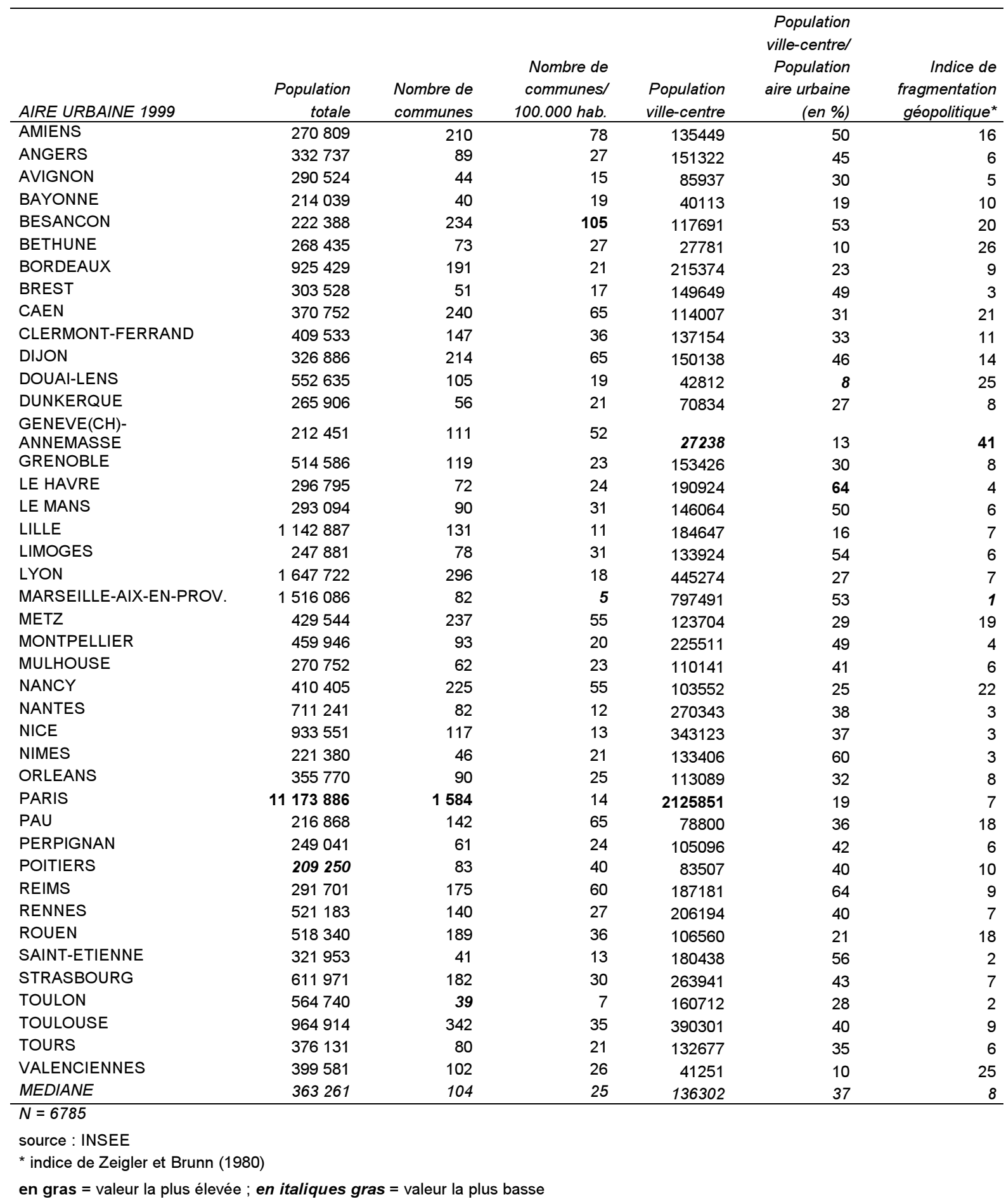


Comme l'indique clairement le tableau 2, le nombre de communes n'est pas proportionnel à la population. Certes, l'aire urbaine de Paris (see Appendix 3) domine largement les autres non seulement pas son poids démographique mais aussi par le nombre d'unités communales qui la composent: 1.584. Mais deux aires urbaines de taille démographique équivalente peuvent enregistrer des niveaux de fragmentation territoriale fort variables : avec plus de 900.00 habitants chacune, Toulouse (342 communes) apparait de ce point de vue trois fois plus fragmentée que Nice (117). De même, avec une population plus réduite de 220.000 habitants, l'aire urbaine de Besançon (234 communes) compte cinq fois plus d'unités que celle de Nîmes (46).

Afin de contrôler la taille démographique, nous avons standardisé cette mesure en calculant pour chaque aire urbaine le nombre de communes pour 100.000 habitants. Si l'on compare les valeurs obtenues avec celles des grandes aires métropolitaines américaines, il apparaît que la fragmentation est plus sensible dans les métropoles françaises dont la valeur médiane est de 25 , soit bien au-dessus des valeurs américaines les plus élevées comme celle de Pittsburgh (17.7 en incluant les municipalités, les townships et les comtés) (voir Altschuler et al. 1999, Orfield 2002). L'usage de cet indicateur nous permet de relativiser le cas de l'aire urbaine de Paris - avec un taux de 14, elle se situe dans la catégorie des aires somme toute moins fragmentées, de même que les deux plus grandes aires qui la suivent, Lyon (18) et Marseille-Aix-en-Provence (5). Besançon atteint à l'autre extrémité un score impressionnant de 105. Souvent, des communes ont une population minuscule, à l'image de Lemenil-Mitry, la plus petite composante de l'aire urbaine de Nancy avec ses 2 habitants, le maire élu depuis 1977 Henri de Mitry, noble descendant de chevaliers du Moyen-Age et propriétaire de deux domaines Le Ménil et Le Mitry, et son épouse... Mais 30 autres communes de cette aire urbaine qui en compte 225 n'ont pas plus de 100 habitants, soit $13 \%$ du total. Cette proportion de très petites unités atteint $18 \%$ dans l'aire urbaine de Besançon. La moitié des 6.785 communes composant nos 42 aires urbaines ont moins de 826 habitants et les trois quarts moins de 2.499 habitants.

Afin de tenir également compte du degré de concentration ou de dispersion de la population à l'intérieur de chaque aire urbaine et donc de la fragmentation non plus seulement territoriale mais également démographique, nous avons complété ce premier indicateur par un 
second mesurant la proportion de la population de la ville-centre par rapport à celle de sa banlieue (voir tableau 2 et figure 2). 


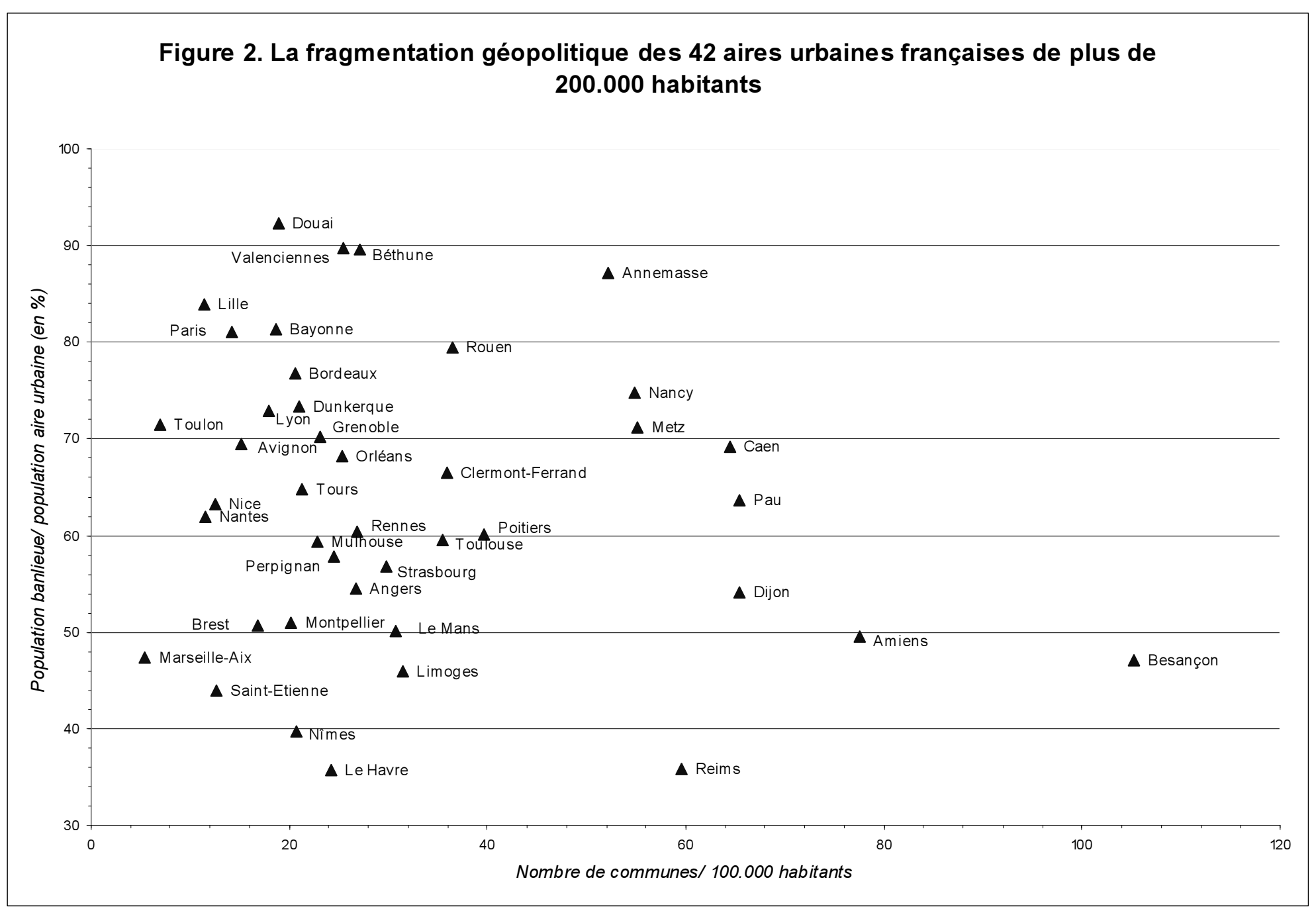


Dans 34 des 42 aires urbaines, la banlieue regroupe aujourd'hui une population supérieure à celle des villes-centres. Le poids démographique de la ville-centre est d'autant plus faible que celle-ci coexiste avec d'autres villes de taille comparable ou substantielle. Il en est ainsi de l'aire de Douai-Lens dont la population de la ville-centre (42.812 habitants) ne concentre que $8 \%$ de la population en raison de la taille concurrente d'un réseau de villes voisines constitué par Lens (36.192 habitants), Liévin (33.463 habitants), Hénin-Beaumont (25.204 habitants) et dix autres municipalités de plus de 10.000 habitants. Une même configuration caractérise les aires urbaines de Béthune (10\% de la population totale), de Valenciennes (10\%), d'Annemasse (13\%), de Lille (16\%), ou de Paris (19\%). Par contre, la ville du Havre (190.924 habitants) attire les deux tiers de la population de son aire urbaine, dont les communes les plus importantes après elle sont par ordre décroissant Montivilliers (16.553 habitants) puis Gonfreville-L'Orcher (9.939 habitants). Une macrocéphalie du même ordre se retrouve à Reims, ville de 187.181 habitants, soit $64 \%$ de la population de son aire urbaine, précédant de loin la deuxième ville de cette aire, Tinqueux qui ne compte que 10.079 habitants.

A partir des deux indicateurs précédents - le nombre de communes/ 100.000 habitants et le pourcentage de la population de la ville-centre par rapport à celle de l'aire urbaine - nous avons mesuré la fragmentation géopolitique de chaque aire urbaine en recourant à l'indice de Zeigler et Brunn (1980). Cet indice est calculé en divisant le nombre de communes pour 10.000 habitants par le pourcentage de la population de la ville-centre par rapport à celle de l'aire urbaine. Il se confirme que les valeurs obtenues - qui s'échelonnent de 1 à MarseilleAix-en-Provence à 41 à Annemasse - sont nettement plus élevées et dispersées que pour les 25 plus grandes aires métropolitaines américaines. Pas moins de 12 aires françaises ont un indice supérieur à l'indice maximal de 12 enregistré aux Etats-Unis pour Pittsburgh (Orfield 2002 : 134). L'utilisation de cet indice montre conduit à réordonner les aires urbaines classées en fonction du critère du nombre de communes : ainsi Besançon qui apparaissait comme l'aire la plus fragmentée territorialement l'est bien moins démographiquement puisque plus de la moitié de sa population (53\%) demeure concentrée dans la ville-centre. Aussi apparaît-elle à travers sa valeur d'indice (20) certes plus fragmentée globalement que la moyenne des aires urbaines mais tout de même moins que celles d'Annemasse (indice de 41), de Béthune (26), de Douai-Lens et Valenciennes (25) ou de Nancy (22). Deuxième constat : les plus grandes aires urbaines ne sont pas les plus fragmentées. Parmi celles de plus de 900.000 habitants, 
seules celles de Bordeaux et de Toulouse affichent un score de 9 légèrement plus élevé que la valeur médiane de 8 . De manière significative, la valeur la plus basse de l'ensemble des aires est atteinte par Marseille-Aix-en-Provence avec 1 - on y compte que 5 communes pour 100.000 habitants, et 53\% vivent à Marseille et 9\% supplémentaires à Aix-en-Provence -, tandis que les valeurs de Paris, Lyon, Lille, et Nice sont respectivement de 8, 7, 7, et 3.

\section{Intermetropolitan Area Comparisons}

Longtemps dominantes dans leur espace d'influence, les villes-centres ont perdu progressivement de leur capacité d'attraction. Leur population a généralement décliné au profit de leurs banlieues en expansion rapide et semble s'être stabilisée entre les deux derniers recensements de 1990 et 1999 (pour plus dé détails: Hoffmann-Martinot 2000). Leur physionomie socio-économique varie sensiblement, contribuant au maintien de fortes disparités entre elles. Nous allons tenter de les comparer entre elles en utilisant les mesures d'urban hardship proposées par Nathan et Adams dans leurs analyses comparatives des métropoles américaines (1976 et 1989).

Rappelons que ces deux auteurs avaient retenu six mesures destinées à appréhender le niveau d'urban hardship dans chaque métropole :

1. le chômage (pourcentage de chômeurs dans la population active)

2. la dépendance (pourcentage de personnes de moins de 18 ans et de plus de 64 ans dans la population totale)

3. l'instruction (pourcentage de personnes de 25 ans ou plus with less than twelfthgrade education)

4. le niveau de revenu (revenu par habitant)

5. le surpeuplement (pourcentage d'unités de logement habitées par plus d'une personne par pièce) $)^{3}$

6. la pauvreté (pourcentage de familles à revenu inférieur à $125 \%$ du niveau de bas revenu)

\footnotetext{
${ }^{3}$ La mesure comparative du surpeuplement dans les villes a été entreprise dès les années 1930 par le Bureau International du Travail à l'échelle européenne, cf. Pinol 2003 (Vol.II : 229s.). Sur l'évolution des statistiques depuis le recensement de 1891 et la baisse tendancielle du surpeuplement des logements à Paris, voir Fijalkow 1998.
} 
Grâce au concours de l'INSEE, du LASMAS-Institut du Longitudinal et à la coopération établie entre le CNRS et l'INSEE, nous avons rassemblé les données issues du dernier recensement de l'INSEE pour les 6.785 communes composant les 42 aires urbaines de plus de 200.000 habitants afin de construire les cinq premières mesures, celle de la pauvreté n'étant pas disponible en France. Pour la mesure de l'instruction, l'équivalent français du niveau inférieur au twelfth grade est le niveau inférieur au baccalauréat. Le niveau de revenu est le revenu médian par habitant issu de l'exploitation par la DGI - Direction Générale des Impôts de la déclaration des revenus 2000 : on doit souligner que cette mesure précise de la richesse municipale est pour la première fois aisément et publiquement accessible en France grâce à la diffusion par l'INSEE en 2003 du CD-ROM Revenus fiscaux des ménages. Année 2000. France métropolitaine par commune. Pour des raisons de confidentialité et de protection de l'anonymat des contribuables, ces données de revenu ne sont pas disponibles pour les communes comptant moins de 50 ménages, si bien qu'en raison de cette limitation plusieurs indicateurs portant non pas sur les villes-centres mais sur leurs banlieues (cf. infra) n'ont pu être calculées que pour 6.273 communes.

Pour chaque mesure retenue, nous avons construit un indice permettant de standardiser les ratios obtenus pour chaque ville entre 0 et 100 à partir de la formule :

$$
\begin{gathered}
X=\left(\frac{Y-Y_{\min }}{Y_{\max }-Y_{\min }}\right) 100 \\
\text { Where }: X \quad=\text { standardized ratio to be created } \\
Y \quad=\text { ratios calculated from data } \\
Y_{\max }=\text { maximum value of } \mathrm{Y} \\
Y_{\min }=\text { minimum value of } \mathrm{Y}
\end{gathered}
$$

Par exemple, pour le ratio de chômage, les valeurs maximale et minimale sont : 24.69 pour Perpignan et 12.03 pour Paris. On attribue la valeur 100 à 24.69 et 0 à 12.03 . Avec $15.68 \%$ de chômage, le ratio standardisé d'une ville comme Angers s'établira par conséquent à : $((15.68-12.03) /(24.69-12.03)) 100=29$.

Prévue pour s'appliquer à des ratios, cette formule a dû cependant être adaptée pour la mesure de revenu qui se présente en Euros par habitant. Nous avons modifié alors la formule de la manière suivante : 


$$
X=\left(\frac{Y-Y_{\max }}{Y_{\min }-Y_{\max }}\right)_{100}
$$

Une fois chacun des indices calculés pour chaque ville, on construit alors un InterCity Composite Index (ICCI) - la moyenne des valeurs des cinq indices - qui permer de classer nos 42 villes dans le tableau 3 . 
Table 3. Intercity Hardship Indexes

\begin{tabular}{|c|c|c|c|c|c|c|}
\hline CENTRAL CITY & $\begin{array}{c}\text { Unemployment } \\
\text { Index }\end{array}$ & $\begin{array}{c}\text { Dependence } \\
\text { Index }\end{array}$ & $\begin{array}{c}\text { Education } \\
\text { Index }\end{array}$ & $\begin{array}{l}\text { Housing } \\
\text { Index }\end{array}$ & $\begin{array}{l}\text { Income } \\
\text { Index }\end{array}$ & $\begin{array}{c}\text { Intercity } \\
\text { Composite } \\
\text { Index }\end{array}$ \\
\hline MARSEILLE & 89 & 77 & 74 & 78 & 87 & 81 \\
\hline PERPIGNAN & 100 & 100 & 81 & 23 & 100 & 81 \\
\hline LE HAVRE & 67 & 80 & 100 & 63 & 92 & 80 \\
\hline DOUAI & 81 & 71 & 78 & 65 & 93 & 78 \\
\hline TOULON & 75 & 99 & 80 & 48 & 82 & 77 \\
\hline AVIGNON & 73 & 76 & 78 & 45 & 100 & 74 \\
\hline NIMES & 85 & 80 & 73 & 31 & 91 & 72 \\
\hline MULHOUSE & 34 & 63 & 89 & 79 & 93 & 72 \\
\hline NICE & 30 & 92 & 67 & 92 & 71 & 70 \\
\hline BETHUNE & 68 & 62 & 82 & 40 & 92 & 69 \\
\hline AMIENS & 63 & 42 & 72 & 72 & 91 & 68 \\
\hline BAYONNE & 61 & 78 & 79 & 24 & 86 & 66 \\
\hline ANNEMASSE & 23 & 50 & 88 & 84 & 80 & 65 \\
\hline DUNKERQUE & 54 & 69 & 81 & 36 & 84 & 65 \\
\hline SAINT-ETIENNE & 39 & 74 & 81 & 42 & 79 & 63 \\
\hline VALENCIENNES & 57 & 54 & 67 & 31 & 85 & 59 \\
\hline LILLE & 63 & 66 & 81 & 15 & 61 & 57 \\
\hline MONTPELLIER & 82 & 22 & 30 & 39 & 88 & 52 \\
\hline REIMS & 32 & 32 & 70 & 29 & 77 & 48 \\
\hline METZ & 18 & 30 & 61 & 41 & 75 & 45 \\
\hline STRASBOURG & 12 & 30 & 45 & 64 & 69 & 44 \\
\hline CAEN & 45 & 25 & 52 & 34 & 62 & 44 \\
\hline PAU & 39 & 52 & 55 & 5 & 64 & 43 \\
\hline BREST & 31 & 43 & 63 & 0 & 74 & 42 \\
\hline ANGERS & 29 & 39 & 59 & 10 & 72 & 42 \\
\hline ORLEANS & 7 & 39 & 49 & 49 & 61 & 41 \\
\hline TOURS & 33 & 34 & 61 & 14 & 62 & 41 \\
\hline ROUEN & 44 & 20 & 48 & 24 & 64 & 40 \\
\hline LIMOGES & 11 & 7 & 38 & 52 & 91 & 40 \\
\hline NANTES & 45 & 33 & 43 & 16 & 61 & 39 \\
\hline BESANCON & 19 & 28 & 52 & 23 & 70 & 39 \\
\hline GRENOBLE & 32 & 23 & 34 & 39 & 64 & 38 \\
\hline LYON & 4 & 38 & 71 & 6 & 58 & 35 \\
\hline $\begin{array}{l}\text { CLERMONT- } \\
\text { FERRAND }\end{array}$ & 18 & 15 & 61 & 12 & 69 & 35 \\
\hline LE MANS & 24 & 34 & 30 & 44 & 39 & 34 \\
\hline BORDEAUX & 55 & 20 & 31 & 12 & 53 & 34 \\
\hline TOULOUSE & 53 & 10 & 27 & 16 & 60 & 33 \\
\hline DIJON & 0 & 21 & 48 & 22 & 48 & 28 \\
\hline POITIERS & 24 & 0 & 43 & 6 & 65 & 28 \\
\hline PARIS & 0 & 16 & 0 & 100 & 0 & 23 \\
\hline RENNES & 10 & 5 & 37 & 9 & 55 & 23 \\
\hline NANCY & 14 & 2 & 22 & 13 & 53 & 21 \\
\hline
\end{tabular}


Pour qu'une ville atteigne un ICCI de 0 , elle devrait enregistrer 0 pour chacun des indices composants, et pour atteindre 100 obtenir également 100 pour chacun des indices.

Le classement obtenu permet de différencier assez clairement les villes-centres prospères de celles qui sont confrontées à d'importantes difficultés socio-économiques. L'ICCI présente un double avantage. Il permet d'abord de prendre en considération une pluralité de dimensions (emploi, dépendance, instruction, logement, revenu) qui ne se recoupent pas nécessairement : une ville globalement aisée comme Paris connaît cependant des problèmes de logement beaucoup plus aigus que dans les autres villes, et une ville en difficulté comme Mulhouse connaît un taux de chômage relativement limité. Cet indice nous permet ensuite d'établir des comparaisons avec d'autres pays où il a déjà été appliqué (EtatsUnis) ou il pourrait facilement l'être (dans plusieurs pays du réseau OIM, tout dépendant de la disponibilité des données).

Le score varie substantiellement entre ses deux valeurs extrêmes, de 21 pour la ville la plus prospère, Nancy, à 81 pour la deuxième ville de France et la plus en difficulté, Marseille. Il est à souligner que la plupart des villes enregistrant l'ICCI le plus élevé appartiennent au Sud-Est du pays - outre Marseille, Perpignan (81), Toulon (77), Avignon (74), Nîmes (72), Nice (70) - et au Nord - Le Havre (80), Douai (78), et Béthune (69).

La capitale Paris appartient aux villes les plus favorisées avec les niveaux les plus bas de toutes les villes françaises de chômage (12\%), de faible instruction (41\% seulement des Parisiens ayant 25 ans et plus ont un niveau d'instruction inférieur au baccalauréat), et de bas revenu (niveau de revenu médian par habitant de $13.731 €$ ). A l'opposé, Perpignan cumule trois records : $25 \%$ de chômeurs, $42 \%$ de personnes dépendantes, et un niveau de revenu médian quasiment deux fois plus faible qu'à Paris avec $7.559 €$ habitant. On constate par conséquent que la situation socio-économique des villes-centres françaises est loin de l'image réductrice tendant à les opposer à leurs homologues américaines : alors que celles-ci seraient globalement rongées par le déclin et la crise urbaine, les villes françaises maintiendraient leur statut de poumon économique et de lieux de concentration des populations les plus favorisées. Le poids toujours écrasant de Paris et de sa région sur le reste du pays (Hoffmann-Martinot 2003) contribue bien entendu à concentrer l'intérêt des médias et de nombreux observateurs sur la capitale lorsqu'ils se penchent sur les particularités et l'évolution des grandes métropoles, ville incontestablement riche et à l'abri des difficultés socio-économiques (même 
si pauvreté et exclusion sociale n'y sont pas absentes). Lorsqu' on y regarde de plus près, le paysage général des villes-centres apparaît beaucoup plus contrasté que ne le laissent entrevoir les zooms habituels sur Paris, ou en province sur des villes bourgeoises traditionnelles comme Lyon ou Bordeaux.

Nous avons ensuite procédé de la même façon pour comparer les banlieues entre elles, à partir des mêmes indicateurs et indice s'appliquant au territoire de l'ensemble de chaque zone suburbaine (Tableau 4).

Contrairement aux variables de chômage, de dépendance, d'instruction et de logement, on ne dispose pas de données sur le revenu médian de l'ensemble du territoire des banlieues. Le revenu médian n'est en effet diffusé par la DGI qu'à l'échelle de chaque commune. Pour chaque banlieue d'aire urbaine, nous avons donc construit et utilisé la variable de substitution suivante :

( $\sum$ (revenu médian municipal $\mathrm{x}$ population municipale) $) /$ population totale des communes composant la banlieue

Le tableau 4 montre que la variation de l'ISCI (Intercity Composite Index) est encore plus forte que celle de l'ICCI puisqu'elle s'échelonne de 92 pour la banlieue de Douai à 19 pour celle d'Annemasse. De même que nos données sur les villes permettent de corriger bon nombre d'approximations diffusées dans les médias voire dans la littérature scientifique, il apparaît que la réalité des banlieues françaises mesurée par l'indice ISCI contredit le cliché couramment répandu dans la presse et bien ancré dans de nombreux esprits selon lequel les banlieues constitueraient en France l'équivalent fonctionnel des villes-centres aux Etats-Unis. En se focalisant sur une certaine catégorie de banlieues en difficulté, on tend à ainsi à sousestimer la grande variété des contextes suburbains et en particulier l'existence voire le développement rapide de banlieues relativement riches et prospères. Les deux tiers des espaces suburbains enregistrent en effet un score ISCI inférieur à 50 qui reflète un niveau relativement faible de difficultés socio-économiques.

En suivant la même approche méthodologique que celle utilisée par Nathan et Adams (1976), nous avons essayé de comparer le classement des villes (ICCI) et de leurs banlieues (ISCI) afin de mesurer l'amplitude des correspondances ou des décalages éventuels. Des 
classements identiques - ville $\mathrm{A}$ et banlieue $\mathrm{A}$ classées en $1^{\text {ère }}$ place, ville B et banlieue B classées en $2^{\text {ème }}$ place, et ainsi de suite - tendraient à indiquer un parallélisme entre le statut relatif des villes et celui de leur environnement. A l'inverse, une non-concordance totale entre les deux classements signifierait une absence d'homologie entre villes-centres et banlieues.

Le tableau 5 présente sur deux colonnes les classements respectifs des villes et de leurs banlieues en distinguant les six sextiles. Dans le tableau 6, nous avons distingué trois types de relations entre les villes et leurs banlieues. The first relationship (Type 1, Significant Disparity) includes cases where there is a difference or two sextiles groups or more between the rankings of the central city and its suburbs on the two interarea indexes. The second relationship (Type 2, Both High) includes cases in which both the central city and its suburbs rank high (having worse conditions) on the two interarea indexes. The third combination (Type 3, Both Low) includes cases in which both the central city and its suburbs rank low on the two indexes. 
Table 4. Intersuburb Hardship Indexes

\begin{tabular}{|c|c|c|c|c|c|c|}
\hline SUBURB & $\begin{array}{c}\text { Unemployment } \\
\text { Index }\end{array}$ & $\begin{array}{c}\text { Dependence } \\
\text { Index }\end{array}$ & $\begin{array}{c}\text { Education } \\
\text { Index }\end{array}$ & $\begin{array}{l}\text { Housing } \\
\text { Index }\end{array}$ & $\begin{array}{c}\text { Income } \\
\text { Index }\end{array}$ & $\begin{array}{c}\text { Intersuburb } \\
\text { Composite } \\
\text { Index }\end{array}$ \\
\hline DOUAI & 94 & 97 & 100 & 73 & 98 & 92 \\
\hline VALENCIENNES & 100 & 85 & 94 & 55 & 100 & 87 \\
\hline BETHUNE & 82 & 92 & 100 & 46 & 87 & 81 \\
\hline DUNKERQUE & 77 & 49 & 87 & 50 & 89 & 70 \\
\hline PERPIGNAN & 73 & 90 & 67 & 20 & 74 & 65 \\
\hline NICE & 63 & 100 & 33 & 83 & 32 & 62 \\
\hline TOULON & 77 & 93 & 45 & 46 & 47 & 62 \\
\hline LILLE & 65 & 63 & 59 & 43 & 58 & 58 \\
\hline AVIGNON & 63 & 60 & 54 & 34 & 65 & 55 \\
\hline SAINT-ETIENNE & 51 & 72 & 66 & 35 & 50 & 55 \\
\hline LYON & 48 & 41 & 82 & 49 & 36 & 51 \\
\hline LE HAVRE & 50 & 39 & 69 & 54 & 43 & 51 \\
\hline AMIENS & 53 & 32 & 71 & 51 & 47 & 51 \\
\hline ROUEN & 61 & 54 & 65 & 31 & 43 & 51 \\
\hline NIMES & 66 & 45 & 48 & 27 & 63 & 50 \\
\hline METZ & 48 & 46 & 68 & 30 & 50 & 48 \\
\hline BAYONNE & 57 & 89 & 41 & 19 & 35 & 48 \\
\hline CAEN & 53 & 34 & 59 & 36 & 50 & 46 \\
\hline ANGERS & 41 & 62 & 52 & 16 & 53 & 45 \\
\hline MARSEILLE-AIX & 73 & 20 & 28 & 60 & 40 & 44 \\
\hline LE MANS & 43 & 53 & 43 & 21 & 52 & 43 \\
\hline TOURS & 48 & 42 & 49 & 20 & 36 & 39 \\
\hline BREST & 37 & 71 & 39 & 0 & 48 & 39 \\
\hline NANTES & 43 & 44 & 47 & 17 & 44 & 39 \\
\hline MULHOUSE & 33 & 40 & 57 & 49 & 10 & 38 \\
\hline NANCY & 47 & 35 & 47 & 21 & 36 & 37 \\
\hline PARIS & 50 & 13 & 15 & 100 & 8 & 37 \\
\hline BESANCON & 33 & 43 & 46 & 19 & 40 & 36 \\
\hline PAU & 42 & 57 & 33 & 7 & 41 & 36 \\
\hline ORLEANS & 36 & 37 & 46 & 34 & 22 & 35 \\
\hline DIJON & 43 & 30 & 42 & 26 & 33 & 35 \\
\hline BORDEAUX & 58 & 16 & 41 & 17 & 36 & 34 \\
\hline REIMS & 39 & 26 & 54 & 22 & 23 & 33 \\
\hline RENNES & 30 & 35 & 38 & 11 & 40 & 31 \\
\hline LIMOGES & 36 & 46 & 37 & 4 & 30 & 31 \\
\hline MONTPELLIER & 59 & 24 & 0 & 31 & 33 & 29 \\
\hline POITIERS & 38 & 19 & 44 & 6 & 38 & 29 \\
\hline STRASBOURG & 30 & 10 & 45 & 42 & 11 & 28 \\
\hline TOULOUSE & 47 & 24 & 15 & 13 & 28 & 25 \\
\hline CLERMONT-FERRAND & 42 & 11 & 39 & 9 & 24 & 25 \\
\hline GRENOBLE & 47 & 12 & 5 & 38 & 22 & 25 \\
\hline ANNEMASSE & 34 & 0 & 19 & 42 & 0 & 19 \\
\hline
\end{tabular}


Table 5. Interarea Hardship Indexes Comparing Cities to Cities and Suburbs to Suburbs

\begin{tabular}{|c|c|c|c|}
\hline Central City & $\begin{array}{c}\text { Intercity } \\
\text { Hardship Index }\end{array}$ & Suburb & $\begin{array}{l}\text { Intersuburb } \\
\text { Hardship Index }\end{array}$ \\
\hline MARSEILLE-AIX-EN-PROV. & 81 & DOUAI-LENS & 92 \\
\hline PERPIGNAN & 81 & VALENCIENNES & 87 \\
\hline LE HAVRE & 80 & BETHUNE & 81 \\
\hline DOUAI-LENS & 78 & DUNKERQUE & 70 \\
\hline TOULON & 77 & PERPIGNAN & 65 \\
\hline AVIGNON & 74 & NICE & 62 \\
\hline NIMES & 72 & TOULON & 62 \\
\hline MULHOUSE & 72 & LILLE & 58 \\
\hline NICE & 70 & AVIGNON & 55 \\
\hline BETHUNE & 69 & SAINT-ETIENNE & 55 \\
\hline AMIENS & 68 & LYON & 51 \\
\hline BAYONNE & 66 & LE HAVRE & 51 \\
\hline ANNEMASSE & 65 & AMIENS & 51 \\
\hline DUNKERQUE & 65 & ROUEN & 51 \\
\hline SAINT-ETIENNE & 63 & NIMES & 50 \\
\hline VALENCIENNES & 59 & METZ & 48 \\
\hline LILLE & 57 & BAYONNE & 48 \\
\hline MONTPELLIER & 52 & CAEN & 46 \\
\hline REIMS & 48 & $\begin{array}{l}\text { ANGERS } \\
\text { MARSEILLE-AIX-EN- }\end{array}$ & 45 \\
\hline METZ & 45 & PROV. & 44 \\
\hline STRASBOURG & 44 & LE MANS & 43 \\
\hline CAEN & 44 & TOURS & 39 \\
\hline PAU & 43 & BREST & 39 \\
\hline BREST & 42 & NANTES & 39 \\
\hline ANGERS & 42 & MULHOUSE & 38 \\
\hline ORLEANS & 41 & NANCY & 37 \\
\hline TOURS & 41 & PARIS & 37 \\
\hline ROUEN & 40 & BESANCON & 36 \\
\hline LIMOGES & 40 & PAU & 36 \\
\hline NANTES & 39 & ORLEANS & 35 \\
\hline BESANCON & 39 & DIJON & 35 \\
\hline GRENOBLE & 38 & BORDEAUX & 34 \\
\hline LYON & 35 & REIMS & 33 \\
\hline CLERMONT-FERRAND & 35 & RENNES & 31 \\
\hline LE MANS & 34 & LIMOGES & 31 \\
\hline BORDEAUX & 34 & MONTPELLIER & 29 \\
\hline TOULOUSE & 33 & POITIERS & 29 \\
\hline DIJON & 28 & STRASBOURG & 28 \\
\hline POITIERS & 28 & TOULOUSE & 25 \\
\hline PARIS & 23 & CLERMONT-FERRAND & 25 \\
\hline RENNES & 23 & GRENOBLE & 25 \\
\hline NANCY & 21 & ANNEMASSE & 19 \\
\hline
\end{tabular}

source : INSEE-DGI 
Table 6. Sixtile Rankings of Cities and Suburbs on the Individual Central City and Suburban Hardship Indexes [a]

\begin{tabular}{|c|c|c|c|c|c|c|c|c|}
\hline & Type 1 & & & Type 2 & & & Type 3 & \\
\hline Metropolitan Area & City & Suburb & Metropolitan Area & City & Suburb & Metropolitan Area & City & Suburb \\
\hline MARSEILLE-AIX & 1 & 3 & PERPIGNAN & 1 & 1 & CAEN & 4 & 3 \\
\hline NIMES & 1 & 3 & LE HAVRE & 1 & 2 & ANGERS & 4 & 3 \\
\hline MULHOUSE & 2 & 4 & DOUAI-LENS & 1 & 1 & PAU & 4 & 5 \\
\hline ANNEMASSE & 2 & 6 & TOULON & 1 & 1 & BREST & 4 & 4 \\
\hline MONTPELLIER & 3 & 6 & AVIGNON & 1 & 2 & NANTES & 5 & 4 \\
\hline REIMS & 3 & 5 & NICE & 2 & 1 & TOURS & 4 & 4 \\
\hline STRASBOURG & 3 & 6 & BETHUNE & 2 & 1 & BESANCON & 5 & 4 \\
\hline LYON & 5 & 2 & AMIENS & 2 & 2 & LIMOGES & 5 & 5 \\
\hline ROUEN & 4 & 2 & BAYONNE & 2 & 3 & GRENOBLE & 5 & 6 \\
\hline LE MANS & 5 & 3 & DUNKERQUE & 2 & 1 & CLERMONT-FER & 5 & 6 \\
\hline NANCY & 6 & 4 & SAINT-ETIENNE & 3 & 2 & ORLEANS & 4 & 5 \\
\hline \multirow[t]{5}{*}{ PARIS } & 6 & 4 & VALENCIENNES & 3 & 1 & DIJON & 6 & 5 \\
\hline & & & LILLE & 3 & 2 & BORDEAUX & 6 & 5 \\
\hline & & & METZ & 3 & 3 & RENNES & 6 & 5 \\
\hline & & & & & & POITIERS & 6 & 6 \\
\hline & & & & & & TOULOUSE & 6 & 6 \\
\hline
\end{tabular}

[a] Rankings are on the basis of the sextile in which each city and suburb fell in the respective intercity and intersuburb hardship indexes. Cities and suburbs coded 1 are in the top sextile, evidencing the highest degree of social and economic hardship.

[b] Type 1 cases are characterized as "Significant Disparity". There was a difference of two sextiles or more between the rankings of the central city and its suburbs on the two interarea indexes.

[c] Type 2 cases are characterized as "Both High". Both the central city and its suburbs ranked in either the third or second or first quintile, implying a relatively high level of hardship for both areas.

[d] Type 3 cases are characterized as "Both Middle Sextile or Below". Both the central city and its suburbs ranked relatively well on the interarea hardship indexes, each falling in either the third, fourth, fifth, or sixth quintile. 
L'observation du Type 1 (Significant Disparity) fait ressortir, parmi les 12 cas, deux types de configurations assez contrastées. Dans les aires urbaines de Marseille, Nîmes, Mulhouse, Annemasse, Montpellier, Reims, et Strasbourg, la ville-centre semble globalement être plus en difficulté que sa banlieue. Certes, il est nécessaire de le rappeler, cette-dernière est globalement considérée alors qu'elle est composée d'une pluralité de communes plus ou moins nombreuses et hétérogènes. A ce modèle s'oppose celui des villes suivantes que sont Lyon, Rouen, Le Mans, Nancy et Paris où la disparité se manifeste dans l'autre sens puisqu'elles se caractérisent par un statut plus favorisé à l'intérieur de leur groupe que leurs banlieues à l'intérieur du leur.

Le Type 2 (Both High) regroupe pour l'essentiel d'anciennes métropoles industrielles $\mathrm{du}$ Nord et du Sud-Est dont aussi bien les villes-centres que leurs banlieues semblent également affectées par les dynamiques de crise et de reconversion économique.

Dans la dernière catégorie, le Type 3 (Both Low) se retrouvent les métropoles relativement aisées où villes et banlieues partagent un profil relativement proche marqué par des conditions de vie et de travail plus favorables que dans la moyenne des métropoles françaises grâce notamment au dynamisme du secteur tertiaire, s'appuyant sur une solide tradition commerciale à Nantes ou à Bordeaux, le développement de nouvelles industries et technologies à Toulouse, où le renforcement de l'innovation scientifique et culturelle à Grenoble ou à Rennes.

\section{Intrametropolitan Area Comparisons}

En utilisant les mêmes 5 indicateurs de chômage, de dépendance, d'instruction, de logement, et de revenu, nous avons cherché à mesurer dans quelle mesure, pour chaque aire urbaine, la ville-centre expérimente plus ou moins de difficultés socio-économiques que sa banlieue, toujours considérée comme une unité globale. Pour cela, nous avons construit un City-Suburb Harship Disparity Index (CSHDI).

Pour chaque mesure retenue, nous avons construit un indice permettant de standardiser les ratios obtenus pour chaque ville entre 0 et 100 à partir de la formule :

$$
X=\left(\underline{Y-Y_{\min }}\right) 100
$$




$$
Y_{\max }-Y_{\min }
$$

Where $: \mathrm{X}=$ standardized ratio to be created

$\mathrm{Y}=$ ratios of city-to-suburb unemployment (or dependence, or education, or housing) rates calculated from data

$\mathrm{Y}_{\max }=$ maximum value of $\mathrm{Y}$

$\mathrm{Y}_{\min }=$ minimum value of $\mathrm{Y}$

Par exemple, pour le ratio ville-banlieue de taux de chômage, les valeurs maximale et minimale sont : 2.16 et 0.85 . On attribue la valeur 100 à 24.69 et 0 à 12.03 . Avec $18.97 \%$ de chômage pour la ville centre et $13.14 \%$ pour la banlieue, le ratio standardisé d'une ville comme Bordeaux s'établira par conséquent à :

$$
[((18,97 / 13,14)-0,85) /(2,16-0,85)] * 100=(0,59 / 1,31) * 100=45
$$

Prévue pour s'appliquer à des ratios, cette formule a dû cependant être adaptée pour la mesure de revenu qui se présente en Euros par habitant. Nous avons modifié alors la formule de la manière suivante :

$$
X=\left(\frac{Y-Y_{\max }}{Y_{\min }-Y_{\max }}\right) 100
$$

Pour chaque ville, nous avons ajouté les valeurs des 5 indicateurs puis les avons divisées par 5. On obtient l'indicateur non ajusté figurant dans le tableau 7. Ainsi, pour Bordeaux :

$$
(45+35+21+28+23) / 5=152 / 5=30.4
$$

Dans une deuxième étape, nous avons ajusté cet index afin de distinguer les villes comparing favorably from those comparing unfavorably to their adjoining suburbs. Like in Nathan and Adams analyses, a composite index score was computed for a hypothetical city that was identical to its suburbs along each of the five indicators.

For such an hypothetical city,

Son indicateur de chômage serait : $((1-0,85) / 1,31) * 100=12$ 
Son indicateur de dépendance serait : $((1-0,781) /(1,077-0,781)) * 100=74$

Son indicateur d'instruction serait : $((1-0,66) /(1,15-0,66) * 100=69$

Son indicateur de logement serait : $((1-0,71) /(1,40-0,71) * 100=42$

Son indicateur de revenu serait : $((1-0,80) /(1,36-0,80) * 100=36$

Son indicateur composite non ajusté serait donc : $(12+74+69+42+36) / 5=233 / 5=47$

Si 47 est ajusté à 100, il suffit ensuite, par une règle de trois, d'ajuster les valeurs non ajustées pour produire le CSHDI ajusté. Nous avons classé les aires urbaines par ordre décroissant de CSHDI (Table 7) et figuré leurs positions respectives sur la figure 3. Dans les aires pour lesquelles la valeur de l'indice est supérieure à 100, la situation de la ville-centre est plus difficile que celle de sa banlieue, et inversement si la valeur est inférieure à 100 .

L'observation des valeurs du CSHDI infirme la thèse selon laquelle les villes-centres seraient dans l'ensemble davantage en difficulté que leurs banlieues mais aussi la thèse inverse. En effet, on observe une répartition à peu près égale entre les 20 villes qui affichent une meilleure «santé socio-économique»que leur périphérie, et les 22 enregistrant au contraire un différentiel qui leur est défavorable.

La comparaison avec les scores des 55 of the largest US SMSA correspondant au même indice (calculé à partir des 5 mêmes indicateurs + celui de pauvreté) révèle deux différences fondamentales entre la France de 1999 et les Etats-Unis de 1980 (Nathan et Adams 1989):

contrairement à leurs homologues françaises, dans la plupart des SMSA (49 sur 55), les hardship conditions sont plus fortes dans les villes que dans leurs banlieues

les valeurs du CSHDI sont nettement plus élevées : elles dépassent 200 dans 24 cas alors que ce niveau n'est atteint nulle part en France, atteignant des niveaux particulièrement élevés à Hartford (536), Newark (509), ou Cleveland (385) 


\begin{tabular}{|c|c|c|c|c|c|c|c|}
\hline AIRE URBAINE 1999 & $\begin{array}{l}\text { Unemployment } \\
\text { Index }\end{array}$ & $\begin{array}{c}\text { Dependence } \\
\text { Index }\end{array}$ & $\begin{array}{l}\text { Education } \\
\text { Index }\end{array}$ & $\begin{array}{l}\text { Housing } \\
\text { Index }\end{array}$ & $\begin{array}{l}\text { Income } \\
\text { Index }\end{array}$ & $\begin{array}{c}\text { Non } \\
\text { Adjusted } \\
\text { Composite } \\
\text { Index }\end{array}$ & $\begin{array}{c}\text { Adjusted } \\
\text { Composite } \\
\text { Index }\end{array}$ \\
\hline ANNEMASSE & 82 & 80 & 100 & 100 & 88 & 90 & 191 \\
\hline MULHOUSE & 100 & 73 & 72 & 77 & 100 & 84 & 180 \\
\hline LE HAVRE & 74 & 93 & 76 & 45 & 65 & 71 & 151 \\
\hline MARSEILLE-AIX & 43 & 100 & 77 & 55 & 61 & 67 & 143 \\
\hline BAYONNE & 53 & 66 & 73 & 51 & 64 & 61 & 130 \\
\hline STRASBOURG & 88 & 50 & 34 & 69 & 61 & 60 & 129 \\
\hline AVIGNON & 49 & 77 & 64 & 54 & 56 & 60 & 127 \\
\hline NIMES & 52 & 90 & 62 & 43 & 45 & 58 & 124 \\
\hline REIMS & 76 & 44 & 55 & 53 & 62 & 58 & 123 \\
\hline PERPIGNAN & 50 & 88 & 58 & 42 & 47 & 57 & 121 \\
\hline AMIENS & 62 & 53 & 47 & 62 & 60 & 57 & 121 \\
\hline LE MANS & 92 & 69 & 73 & 25 & 19 & 56 & 119 \\
\hline TOULON & 29 & 85 & 72 & 37 & 48 & 54 & 115 \\
\hline SAINT-ETIENNE & 47 & 70 & 59 & 46 & 41 & 53 & 112 \\
\hline MONTPELLIER & 63 & 34 & 45 & 51 & 69 & 52 & 111 \\
\hline BREST & 82 & 36 & 57 & 46 & 37 & 51 & 110 \\
\hline NICE & 19 & 75 & 66 & 40 & 46 & 49 & 105 \\
\hline PAU & 73 & 53 & 52 & 38 & 31 & 49 & 104 \\
\hline LIMOGES & 53 & 42 & 67 & 51 & 32 & 49 & 104 \\
\hline ORLEANS & 55 & 47 & 38 & 61 & 42 & 49 & 103 \\
\hline BESANCON & 82 & 32 & 41 & 47 & 38 & 48 & 102 \\
\hline CLERMONT-FERRAND & 51 & 31 & 55 & 51 & 51 & 48 & 102 \\
\hline GRENOBLE & 50 & 41 & 47 & 37 & 46 & 44 & 94 \\
\hline TOULOUSE & 69 & 20 & 32 & 49 & 36 & 41 & 88 \\
\hline NANTES & 76 & 37 & 31 & 37 & 25 & 41 & 88 \\
\hline ANGERS & 66 & 35 & 44 & 27 & 30 & 40 & 86 \\
\hline METZ & 35 & 33 & 38 & 56 & 37 & 40 & 84 \\
\hline TOURS & 50 & 39 & 49 & 25 & 32 & 39 & 83 \\
\hline POITIERS & 70 & 10 & 33 & 41 & 34 & 38 & 80 \\
\hline RENNES & 86 & 9 & 29 & 33 & 22 & 36 & 76 \\
\hline CAEN & 48 & 33 & 34 & 31 & 22 & 33 & 71 \\
\hline DUNKERQUE & 18 & 75 & 47 & 12 & 11 & 33 & 69 \\
\hline $\begin{array}{l}\text { BETHUNE } \\
\text { BORDFAUX }\end{array}$ & 20 & 48 & 42 & 25 & 22 & 31 & 67 \\
\hline BORDEAUX & 45 & 35 & 21 & 28 & 23 & 30 & 65 \\
\hline DIJON & 31 & 29 & 39 & 29 & 20 & 30 & 63 \\
\hline DOUAI-LENS & 16 & 54 & 38 & 22 & 13 & 29 & 61 \\
\hline ROUEN & 33 & 18 & 27 & 23 & 29 & 26 & 55 \\
\hline LILLE & 5 & 0 & 20 & 48 & 50 & 24 & 52 \\
\hline LYON & 41 & 39 & 2 & 26 & 11 & 24 & 50 \\
\hline $\begin{array}{l}\text { NANCY } \\
\text { PABIS }\end{array}$ & 35 & 5 & 9 & 20 & 24 & 19 & 40 \\
\hline PARIS & 16 & 31 & 0 & 30 & 0 & 16 & 33 \\
\hline VALENCIENNES & 0 & 42 & 30 & 0 & 3 & 15 & 32 \\
\hline
\end{tabular}

source : INSEE-DGI 
Figure 3. City Suburb Hardship Disparity Index

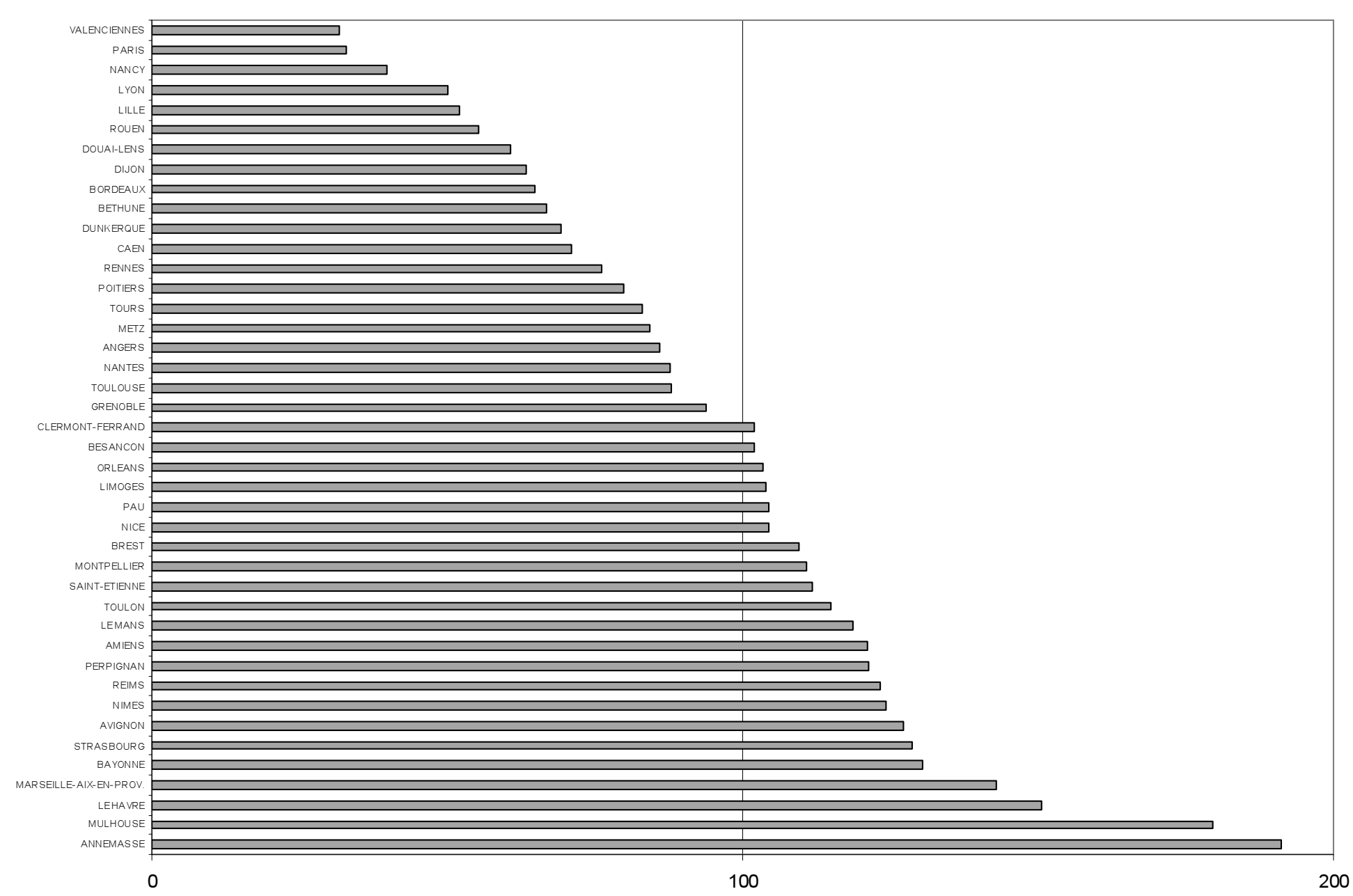


En-dehors des groupes situés aux extrêmes - celui des villes défavorisées par rapport à leur banlieue comme Annemasse, Mulhouse, et Le Havre, et celui des villes favorisées par rapport à leur banlieue comme Valenciennes, Paris, et Nancy - le tableau 7 et la figure 3 révèlent qu'une grande partie des aires urbaines françaises enregistrent un score de CSHDI proche de 100. Globalement, les disparités entre le ville et sa périphérie apparaissent donc modérées. Certes, mais les indices utilisés gomment les disparités potentielles existant entre les différentes communes périphériques. Or, il est indispensable de se pencher sur les caractéristiques éminemment variables des différentes mosaïques suburbaines afin d'échapper au risque de généralisation excessive tendant à considérer l'espace périphérique comme relativement homogène. L'hétérogénéité démographique et socio-économique des banlieues est largement sous-estimée et inexplorée dans de nombreux pays. De récents travaux comme celui de l'urbaniste Hayden (2003) ou des politologues Dreier et al. (2001) permettent de réévaluer et de différencier l'image communément admise et répandue aux Etats-Unis de standardized places réservées aux classes moyennes Wasp. D’une autre nature, la complexité qui caractérise les banlieues françaises est au moins aussi importante. Il suffit pour cela d'examiner la distribution de notre indicateur de richesse municipale (le revenu médian par habitant) à l'intérieur de chacune de nos 42 aires urbaines.

La figure 4 représente cette distribution par aire urbaine dans les 6.273 communes pour lesquelles le revenu médian par habitant est publié et diffusé par la Direction Générale des Impôts (DGI). Nous avons représenté cette distribution par une ligne reliant les valeurs minimale et maximale, indiquant les différents quartiles ainsi que par un rond plein et foncé la position de la ville centre. Ainsi, pour l'aire urbaine d'Amiens, le revenu médian par habitant le plus faible est celui de la commune de Saint-Ouen avec $6.675 €$, le plus élevé celui de Saint-Fuscien avec $15.591 €$, le premier quartile se situe à $8.319 €$, la médiane à $9.255 €$, le troisième quartile à $10.022 €$, et la ville-centre, Amiens, à $8.118 €$.

-- NEXT PAGE : Figure 4. Distribution of Municipal Median Income per Capita in $€(2000)--$ 
$\times$ Minimum $\Delta$ 1st quartile - Median $\diamond$ 3rd quartile $*$ Maximum $\bullet$ Central City

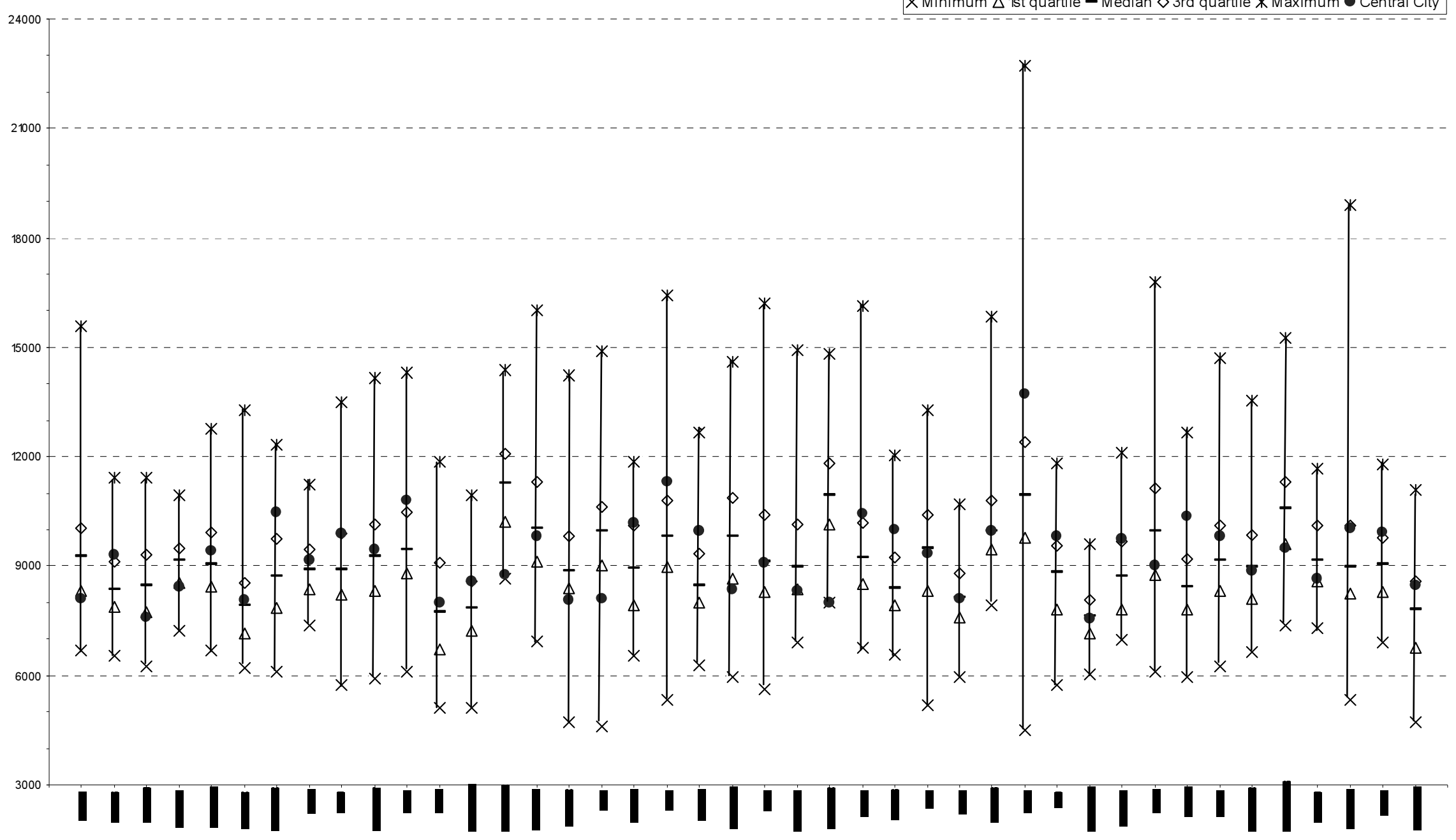


Parmi les éléments d'observation les plus marquants, relevons la grande variation de la distribution du revenu médian d'une aire urbaine à l'autre. Une relative homogénéité caractérise de ce point de vue les aires urbaines de Bayonne - où les valeurs s'étagent de $7.227 €$ à Biarrotte à $10.943 €$ à Bassussarry - ou de Perpignan - de $6037 €$ à Opoul-Perillos à $9.603 €$ à Cabestany - mais le niveau de concentration est sans doute favorisé par le nombre relativement faible de communes qui les composent. A l'inverse, l'aire urbaine de Paris connaît l'écart le plus important et le plus impressionnant entre des communes urbaines de la France « une et indivisible »: le revenu médian par habitant est tout simplement cinq fois plus faible à Clichy-sous-Bois (4.482 €) qu'à Neuilly-sur-Seine (22.731€), deux villes qu sont respectivement la plus pauvre et la plus riche à l'échelle de l'ensemble des 42 aires urbaines. Bien que moindre, le ratio de 3.5 est également substantiel dans l'aire urbaine de Toulouse entre Auribail (5.338 €) et Vieille-Toulouse (18.886€).

Deuxième constat : la médiane municipale varie assez régulièrement autour de $9.000 €$ dans la plupart des aires urbaines, s'établissant au-dessous de $8.000 €$ seulement à Béthune (7.907 €), Douai-Lens (7.738 €), Dunkerque (7.850€), Perpignan (7.636€) et Valenciennes (7.813 €), et dépassant $10.000 €$ uniquement dans les aires de Annemasse (11.275 €), Grenoble (10.020€), Mulhouse (10.939€), Paris (10.945€), et Strasbourg (10.586€).

Les niveaux les plus élevés de pauvreté - que nous pouvons observer sur la figure 4 au-dessous du niveau de $6.000 €$ - et de richesse - au-dessus de $15.000 €$ - ne se trouvent pas dans les villes-centres mais dans les banlieues. Les différences socio-économiques apparaissent considérables entre les banlieues favorisées et défavorisées, comme en témoignent trois paires de communes les plus riches et les plus pauvres au sein de leurs aires urbaines respectives à partir de nos cinq indicateurs de chômage (IC : taux de chômage), de dépendance (ID : \% personnes de moins de 18 ans ou de plus de 64 ans dans la population municipale totale), d'instruction (II : \% de personnes de 25 ans et plus ayant un niveau d'instruction inférieur au baccalauréat), de logement (IL : \% de logements occupés par plus d'une personne par pièce), et de revenu (IR : revenu médian par habitant) :

AIRE URBAINE DE PARIS

\begin{tabular}{lccccr} 
& $I C$ & $I D$ & $I I$ & $I L$ & \multicolumn{1}{c}{$I R$} \\
Clichy-sous-Bois & 24 & 41 & 80 & 34 & 4.482 \\
Neuilly-sur-Seine & 9 & 40 & 28 & 9 & 22.731
\end{tabular}


AIRE URBAINE DE LYON

\begin{tabular}{lrrrrr} 
& $I C$ & $I D$ & $I I$ & \multicolumn{1}{c}{$I L$} & \multicolumn{1}{c}{$I R$} \\
Vaulx-en-Velin & 23 & 39 & 82 & 19 & 5.342 \\
Saint-Didier-au-Mont-d'or & 7 & 38 & 39 & 6 & 16.404
\end{tabular}

AIRE URBAINE DE METZ

\begin{tabular}{lcccrr} 
& $I C$ & $I D$ & $I I$ & \multicolumn{1}{c}{$I L$} & \multicolumn{1}{c}{$I R$} \\
Woippy & 23 & 37 & 82 & 17 & 5.607 \\
Sainte-Ruffine & 6 & 39 & 47 & 1 & 16.206
\end{tabular}

Les villes-centres sont-elles plus riches que les communes qui les entourent? Oui et non. La relation entre ville et banlieues ne peut être ramenée à un modèle unique. En effet, dans 19 aires urbaines sur 42, soit un peu moins que la moitié des cas, la ville-centre appartient à la moitié la moins riche des communes de son aire. Dans le premier quartile - soit le quart des communes les moins riches - figurent pas moins de 10 villes-centres : Amiens, Avignon, Bayonne, Annemasse (la troisième commune la plus pauvre de son aire avec 8.767 $€)$, Le Havre, Lille, Marseille, Montpellier, Mulhouse (la commune la plus pauvre de son aire avec $8.003 €$ ), et Strasbourg. On trouve ensuite 9 villes-centres dans le second quartile : Grenoble, Metz, Nice, Nîmes, Orléans, Perpignan, Reims, Saint-Etienne, et Toulon. Les autres villes se répartissent pour 9 d'entre elles dans le troisième quartile (Besançon, Béthune, Brest, Caen, Clermont-Ferrand, Douai, Dunkerque, Toulouse et Valenciennes), et pour 14 dans le quatrième quartile regroupant les communes les plus aisées (Angers, Bordeaux, Dijon, Limoges, Lyon, Le Mans, Nancy, Nantes, Paris, Pau, Poitiers, Rennes, Rouen, et Tours).

Paris est incontestablement une ville globalement prospère, en tout cas la plus riche des villes-centres avec un revenu médian de $13.731 €$ par habitant, suivie de Lyon (11.308 €), puis de Dijon (10.799 €). Mais, à l'intérieur de son aire urbaine, on compte pas moins de 183 banlieues dont le revenu médian dépasse le niveau déjà élevé de la capitale, et même souvent de façon substantielle. En-dehors de Neuilly, les exemples sont nombreux de banlieues peuplées de manière dominante par les classes les plus favorisées, comme Saint-Nom-LaBretèche, Le Vesinet, Saint-Cloud, Sceaux, Jouy-en-Josas, ou Maisons-Laffitte. 
Les différentes données socio-démographiques que nous avons présentées et commentées jusqu'à présent nous permettent d'établir quelques constats principaux :

on a assisté depuis surtout les années 1970 à une extension continue des aires urbaines dont la croissance a profité surtout aux communes périurbaines au détriment des villes centres et de leurs banlieues immédiates

le modèle français d'étalement urbain partage des caractéristiques communes avec le modèle des aires métropolitaines américaines tant dans ses causes qu'à travers ses conséquences. Ainsi, alors que la suburbanisation des années 1950-60 a été largement guidée et favorisée par des programmes étatiques de desserrement des villes-centres (politique des grands ensembles, création des villes nouvelles dans la région parisienne, ZUP), le mouvement amorcé à parti des années 1980 correspond, comme aux Etats-Unis, non pas à une politique top-down mais à des stratégies beaucoup plus privées et individualisées. Pour des raisons à la fois économiques et liées à leurs aménités historiques et culturelles, les villes-centres demeurent cependant en France plus qu'aux Etats-Unis des pôles d'attraction forts qui ont empêché un mouvement massif de fuite des classes moyennes vers la périphérie (pour une approche théorique comparative basée sur les aménités: Brueckner et al. 1999). Le modèle urbain français demeure donc plus monocentrique.

à une échelle nationale et globale, les disparités socio-économiques entre les villes-centres et leurs banlieues apparaissent relativement limitées et plus différenciées qu'aux Etats-Unis, ainsi que l'attestent la comparaison francoaméricaine des indices de Nathan et Adams et les mesures de richesse classiques telles que le revenu médian par habitant

il existe néanmoins des variations importantes des profils municipaux tant entre les aires urbaines qu'entre les communes appartenant à une même aire urbaine, rendant indispensable une exploration approfondie de la relation entre la ville-centre et ses banlieues

\section{To what extent are citizens political attitudes determined by their commune of residence?}


Notre hypothèse centrale est que le mouvement de suburbanisation qui s'est développé en France en gros depuis les années 1970 s'apparente dans sa structure et dans ses conséquences sur les attitudes politiques des citoyens à la tendance générale observée aux Etats-Unis. Pour reprendre les termes de Putnam (2000 : 209) :

"As suburbanization continued, however, the suburbs themselves fragmented into a sociological mosaic - collectively heterogeneous but individually homogeneous, as people fleeing the city sorted themselves into more and more finely distinguished "lifestyle enclaves ", segregated by race, class, education, life stage, and so on ».

Cette tendance à la ségrégation résidentielle a été davantage observée et étudiée à l'échelle des quartiers d'une grande ville qu'entre les communes et notamment entre celles qui ont connu la croissance démographique la plus importante au cours de ces dernières années, les communes périurbaines. Mais plusieurs études récentes confirment que la polarisation économique et sociale de l'espace urbain français ne cesse de s'accroître depuis les années 1980 (voir notamment : Bessy 1998 ; Chenu et Tabard 1994, Rouxel 2003).

De nombreuses récentes communes suburbaines attirent en majorité des membres des nouvelles classes moyennes, les «néo-périurbains», souvent de jeunes parents avec des enfants en bas âge, en quête d'espace et de ruralité, de confort de logement, de sécurité (l'enquête permanente Conditions de vie - Vie de quartie réaliser par l'INSEE révèle que le sentiment d'insécurité est beaucoup moins élevé dans ce type de communes que dans les communes anciennes à forte concentration d'habitat collectif : Le Toqueux 2003), de distance suffisante par rapport à la grande ville bruyante, compacte, et stressante. Bref, échapper à tout prix à l'engrenage «métro, boulot, dodo». La qualité de vie, un certain retour à une campagne idéalisée mais pas trop éloignée de la ville et de ses opportunités d'emploi grâce à la proximité de réseaux de transports routiers, autoroutiers et ferroviaires, le coût du logement et notamment l'accès plus facile à la propriété, le niveau plus avantageux de pression fiscale locale constituent autant de facteurs qui expliquent la périurbanisation contemporaine. Plusieurs études récentes documentent ces stratégies de localisation résidentielle dans les communes périurbaines et de dissociation croissante entre de lieux d'habitat et d'emploi (sur l'aire urbaine de Lyon: Andan et al. 1999). L'humoriste Alphonse Allais était donc prophétique lorsqu'il énonçait au XIXème siècle : «Les villes devraient être construites à la campagne. L'air y est tellement plus pur »! Exploitant les enquêtes Logement de l'INSEE, Cavailhès et Goffette-Nagot (2003) la loi de l'économie urbaine selon laquelle les ménages 
font un arbitrage entre le coût des déplacements domicile-travail et le coût du logement. Pour qu'ils soient indifférents à habiter un peu plus près ou un peu plus loin du centre, il faut que si le coût du déplacement s'accroît, c'est-à-dire si la distance augmente, la rente foncière baisse. Ils montrent clairement, en étudiant l'ensemble des aires urbaines françaises, que plus la distance au centre augmente, plus la part des maisons individuelles et de la surface habitable tendent à croître en raison de la diminution de la rente foncière. En distinguant quatre catégories de communes - communes-centres, communes de banlieue, périurbain en deçà de $20 \mathrm{~km}$, et périurbain au-delà de $20 \mathrm{~km}$ - leur étude montre enfin qu'en 1996 le revenu moyen des ménages pour l'ensemble des aires urbaines françaises était le plus élevé dans les communes périurbaines et que la progression la plus forte depuis 1984 était enregistrée dans le périurbain au-delà de $20 \mathrm{~km}(+85 \%)$.

Le « retour à la nature » comme antidote à la grande ville pernicieuse et dangereuse, véritable lieu de perdition a, comme on le sait, fortement influencé le mouvement de suburbanisation aux Etats-Unis. Cette aspiration à fort soubassement chrétien est également très présente en France où les origines catholiques et paysannes de la majeure partie de la population favorisent un rapport de défiance voire de rejet à l'égard de la grande ville et de son «amoralité » et une volonté de s'en distancier autant que possible grâce au développement des transports individuels et collectifs. Comme le soulignent Sauvage et al. (2002 : 56) à travers les transformations de l'aire urbaine de Rennes,

"le monde de l'origine rurale a longtemps été perçu, représenté, rêvé comme le paradis originel ; l'obligation de venir en ville fut, pour de nombreux contingents de ruraux, l'occasion de se joindre aux files des nouveaux urbains destinés à s'établir dans "l'enfer des grands ensembles »... »

La forme urbaine, le type d'habitat et la composition sociale des banlieues se sont régulièrement différencié depuis leur développement rapide dans la deuxième moitié du XIXème siècle, que nous illustrerons à travers quatre banlieues aux profils bien contrastés de l'aire urbaine de Bordeaux (cf. Table 8). Autour de la ville centre s'est d'abord constituée une première couronne de banlieues dont la densité bâtie tend à augmenter; une partie d'entre elles, de tradition industrielle, conservent un appareil productif tout en se tertiarisant. La banlieue industrielle et populaire jouxtant Bordeaux en est un exemple. $60 \%$ de ses logements furent construits avant 1967, 74\% avant 1975, souvent des pavillons pour ouvriers et employés, l'habitat collectif représentant $37 \%$ du total des logements. Banlieue donc 
ancienne, longtemps seul bastion communiste de l'agglomération, elle connait depuis la fin des années 1980 une certaine forme de gentrification attirant à présent les classes moyennes en quête de maisons individuelles et de jardins à un coût plus abordable qu'à Bordeaux (on compte seulement $30 \%$ de propriétaires à Bordeaux, contre $49 \%$ à Bègles). La progressive diversification sociale et culturelle de cette banlieue provoquée par la migration de ces nouvelles couches sociales explique en grande partie la transition en 1989 d'un communisme muncipal traditionnel à un new politics leadership incarné par le médiatique député écologiste Noël Mamère.

\begin{tabular}{|c|c|c|c|c|c|c|c|c|}
\hline & Population & $\begin{array}{r}\text { Housing } \\
\text { Units }\end{array}$ & $\begin{array}{r}\% \text { built } \\
\text { before } \\
1949\end{array}$ & $\begin{array}{r}\text { \% built } \\
1949- \\
1974\end{array}$ & $\begin{array}{r}\% \text { built } \\
\text { since } \\
1975\end{array}$ & $\begin{array}{r}\% \\
\text { Collective } \\
\text { Buildings }\end{array}$ & $\begin{array}{l}\text { Median } \\
\text { Income }\end{array}$ & $\begin{array}{r}\% \\
\text { Housing } \\
\text { Owners }\end{array}$ \\
\hline Bègles & 22.538 & 10.317 & 37 & 37 & 26 & 37 & 9.332 & 49 \\
\hline Lormont & 21.340 & 8.646 & 10 & 59 & 31 & 71 & 6.082 & 30 \\
\hline Le Haillan & 8.134 & 3.142 & 8 & 29 & 63 & 19 & 11.240 & 63 \\
\hline Martignas/Jalle & 5.581 & 1.848 & 4 & 15 & 81 & 2 & 11.109 & 83 \\
\hline
\end{tabular}

source : INSEE

C'est dans cette même première couronne qu'ont été construits dans les années 1960 les grands ensembles immobiliers sociaux à la désormais triste réputation dans le cadre de l'urbanisme opérationnel et des zones à urbaniser en priorité (ZUP). Ces opérations, destinées notamment à accueillir les enfants du baby boom, les vagues de migrants d'origine rurale mais aussi les rapatriés d'Algérie ont été concentrées sur des programmes massifs, $60 \%$ des ZUP construites en France offrant une capacité d'accueil comprise entre 2000 et 5000 logements. Comme le souligne Burgel (in : Pinol 2003 : 690), certaines sont entrées dans la légende des quartiers qualifiés plus récemment de difficiles: Lyon-La Duchère (1958, 6.000 logements), Caen-Hérouville (1962, 8.000 logements), Nantes-Saint-Herblain (1962, 7.500 logements), Marseille-ZUP Nord n³ (1964, 25.000 logements), ou Toulouse-Le Mirail (1964, 23.000 logements). Dans l'agglomération de Bordeaux, Lormont appartient à cette catégorie de banlieues caractérisées par la construction de tours et de barres massives de logements sociaux dans les années 1960-70 : 59\% de ses logments actuels ont été construits en l'espace de seulement onze ans, soit entre 1968 et 1981. Plus tardivement urbanisée que Bègles, Lormont concentre aujourd'hui de nombreux problèmes économiques et sociaux et enregistre, parmi toutes les communes de l'aire urbaine, les taux les plus bas de richesse 
municipale (6.082 € par personne de revenu médian) et de propriétaires (30\%), et une forte proportion d'immigrés, près d'un habitant sur cinq.

A l'ouest de l'agglomération bordelaise, Le Haillan présente un profil urbanistique et socio-démographique tout à fait différent de celui de Lormont. Comme celle-ci, la commune du Haillan est longtemps resté un petit bourg rural peuplé de quelques maraîchers : 8\% seulement des logements existants furent construits avant 1949, et son développement est encore plus récent puisqu'il date surtout des années 1980-90;63\% des logements ont été construits depuis 1975. Ici, les logements collectifs sont limités (19\%) au profit des pavillons et de l'habitat individuel. La commune attire surtout des cadres moyens et supérieurs, notamment du pôle aéronautique voisin, dotés d'un patrimoine suffisant pour acquérir niveau de revenu médian de $11.240 €$, soit pratiquement le double de celui de Lormont, et taux de $63 \%$ de propriétaires - plus souvent que louer des maisons spacieuses avec jardins ou parcs. Le prix élevé du foncier résulte du niveau élevé de qualité de vie résultant du dynamisme économique, de très bonnes liaisons avec le cœur de l'agglomération, de la proximité des lacs, du Médoc et du littoral atlantique, du bon tissu de commerces de proximité, d'infrastructures culturelles et d'équipements sportifs.

La commune de Martignas-sur-Jalles est une banlieue encore plus jeune dont $81 \%$ des logements ont été construits depuis 1975, presque tous des maisons individuelles $(98 \%$ du parc immobilier) et où le pourcentage de propriétaires est l'un des plus élevés de l'aire urbaine $(83 \%)$. Comme Le Haillan, ses responsables misent sur ses atouts de qualité de vie et ses aménités pour attirer une population jeune (moyenne d'âge de 35 ans), qualifiée et aisée (11.109 € de revenu médian) à la recherche d'espace (avec 2.639 hectares, le territoire très boisé est particulièrement étendu puisqu'il représente $53 \%$ de la superficie de la ville de Bordeaux). On peut lire sur le site Internet de la municipalité la «publicité » suivante (http://www.martignas.org/):

" A 18 kilomètres à l'ouest de Bordeaux, à mi-temps des quais de la capitale aquitaine et de l'océan Atlantique, Martignas-sur-Jalle présente tous les atouts d'une ville à la campagne. L'odeur des pins des Landes qui lui servent d'écrin, la douceur de l'air marin, le calme des bords de Jalle offrent aux Martignassais un cadre forestier où il fait bon vivre, à quelques pas d'une des grandes métropoles françaises ».

Le développement récent de communes périurbaines telles que Martignas confirme en France une tendance de fond observable non seulement aux Etats-Unis mais également dans 
d'autres pays industrialisés. La suburbanisation dépend de plus en plus non pas de contraintes exercées par de grands agents publics (Etat) ou privés (entreprises), mais de stratégies individuelles de migration et de localisation de la part de ménages guidés par les arbitrages auxquels ils procèdent en fonction de leurs préférences résidentielles.

Historiquement, comme le soulignent Thisse et al. (2003), la répartition des populations et des activités au sein des villes a, pendant longtemps, été dictée par des logiques non économiques. Les règles d'affectation du sol y étaient principalement régies par la coutume, la religion ou le pouvoir politique, ce qui, dans certains cas, pouvait favoriser une plus grande mixité sociale au sein des mêmes quartiers, voire dans les mêmes logements. Dans ces sociétés, toutefois, la proximité spatiale allait souvent de pair avec une distance sociale très grande entre groupes sociaux et individus.

Lorsque émergèrent la propriété privée des sols et le droit de les aliéner, l'achat et la vente de parcelles conduisirent à une spécialisation croissante des localisations fondée sur leur rentabilité relative. En bref, l'existence de marchés fonciers est devenue un élément structurant de l'espace urbain qui conduit à une séparation progressive des lieux de travail, de résidence et de loisir.

Le principe de base expliquant la formation du prix du sol - la rente foncière - et l'organisation des activités au sein de l'espace urbain en fonction des variations de ce prix a été énoncé dès 1826 par von Thünen dans son étude de la rente agricole. Ce principe reste valable pour les sols urbains : tout se passe comme si, de manière implicite, chaque ménage comparait toutes les localisations possibles et évaluait pour chacune d'elle le montant maximum (ci-après appelé « enchère ») qu'il serait disposé à payer pour y résider. Chaque lot est alors attribué au ménage ayant l'enchère la plus élevée pour le lot en question. L'enchère d'un ménage pour une localisation particulière dépend de nombreux facteurs tels que la qualité du logement et de son voisinage immédiat, les facilités existantes à proximité et les moyens de transport disponibles. La distance au centre d'emploi où doivent se rendre les membres du ménage reste un des facteurs fondamentaux, même si son rôle décline avec les progrès réalisés dans le domaine du transport.

Le logement étant un bien essentiel dont la consommation augmente avec le revenu, quand on passe d'une catégorie socio-économique à une autre plus riche, les ménages ont 
tendance à être plus éloignés du centre-ville afin de pouvoir consommer de plus grandes surfaces. Dans ce cas, ce seraient les ménages les plus pauvres qui s'installeraient près du centre-ville et y utiliseraient de petites surfaces, tandis que les ménages les plus riches devraient résider en périphérie, phénomène effectivement observé dans la plupart des villes américaines, mais également dans des pays d'Europe. Il n'est pas inutile d'insister sur le fait que cette stratification est le résultat d'une concurrence pour l'occupation du sol entre utilisateurs possédant des disponibilités à payer différentes; il n'est en rien le produit de mécanismes de discrimination, quand bien même ceux-ci peuvent, dans la réalité, venir la renforcer.

La baisse du coût des migrations alternantes, jointe à la hausse des revenus des ménages, constitue la raison majeure de l'étalement caractéristique des villes modernes, y compris en France, beaucoup moins compactes que les villes anciennes (urban sprawl).

Les choix de localisation des jeunes ménages - qui tendent à se sédentariser après l'âge approximatif de 45 ans - résultent donc d'un arbitrage entre leurs revenus disponibles et la recherche d'un niveau optimal d'aménités locales. Dans de nombreuses aires métropolitaines des Etats-Unis, le niveau général des aménités disponibles s'est considérablement détérioré dans les villes-centres, contribuant à l'exode massif des catégories supérieures. En revanche, en France, une bonne partie des villes-centres ont longtemps maintenu un niveau élevé d'aménités (patrimoine historique, écoles, institutions culturelles, restaurants, spectacles,...) leur permettant de maintenir ou d'attirer sur leur territoire une proportion nettement plus élevée de cadres moyens et supérieurs (voir la comparaison éclairante US-Allemagne-France de Sellers 1999). Mais la croissance au cours des dernières années d'une série de désaménités dans les villes-centres françaises - parc immobilier insuffisant et devenu de plus en plus coûteux, problèmes devenus aigus de circulation et de stationnement automobile, pollution croissante dont les effets dangereux sur la santé sont de plus en plus connus et vulgarisés, insécurité perçue comme en augmentation sensible, ... - a incité une proportion croissante de jeunes ménages à s'installer à une distance suffisante du centre pour acquérir une maison dans une commune périurbaine. Plus le différentiel d'aménités naturelles (topographie), historiques (patrimoine architectural et culturel), et économiques et socio-culturelles (commerces, écoles, institutions culturelles) tend à être déséquilibré au détriment de la ville-centre, plus les ménages à statut économique et culturel élevé auront tendance à quitter la ville-centre. 
Cette périurbanisation semble suivre le «modèle US » dans la mesure où elle semble accentuer la ségrégation socio-économique des populations municipales. Nous l'avons vu à travers l'examen des profils du Haillan et de Martignas, deux banlieues parmi les plus récentes et les plus aisées de l'aire urbaine de Bordeaux. Les motifs d'installation et les caractéristiques de leurs habitants illustrent cette tendance à la constitution d'enclaves favorisées. Ces ménages ont les moyens d'acquérir des terrains et des maisons spacieuses bien reliées au centre d'emploi principal de Bordeaux, et de payer un ticket d'entrée qui exclut de facto les groupes sociaux moins favorisés. Il n'est donc pas surprenant que la variation des revenus soit moins importante dans les communes périurbaines que dans les autres communes des aires urbaines (Caruso 2003).

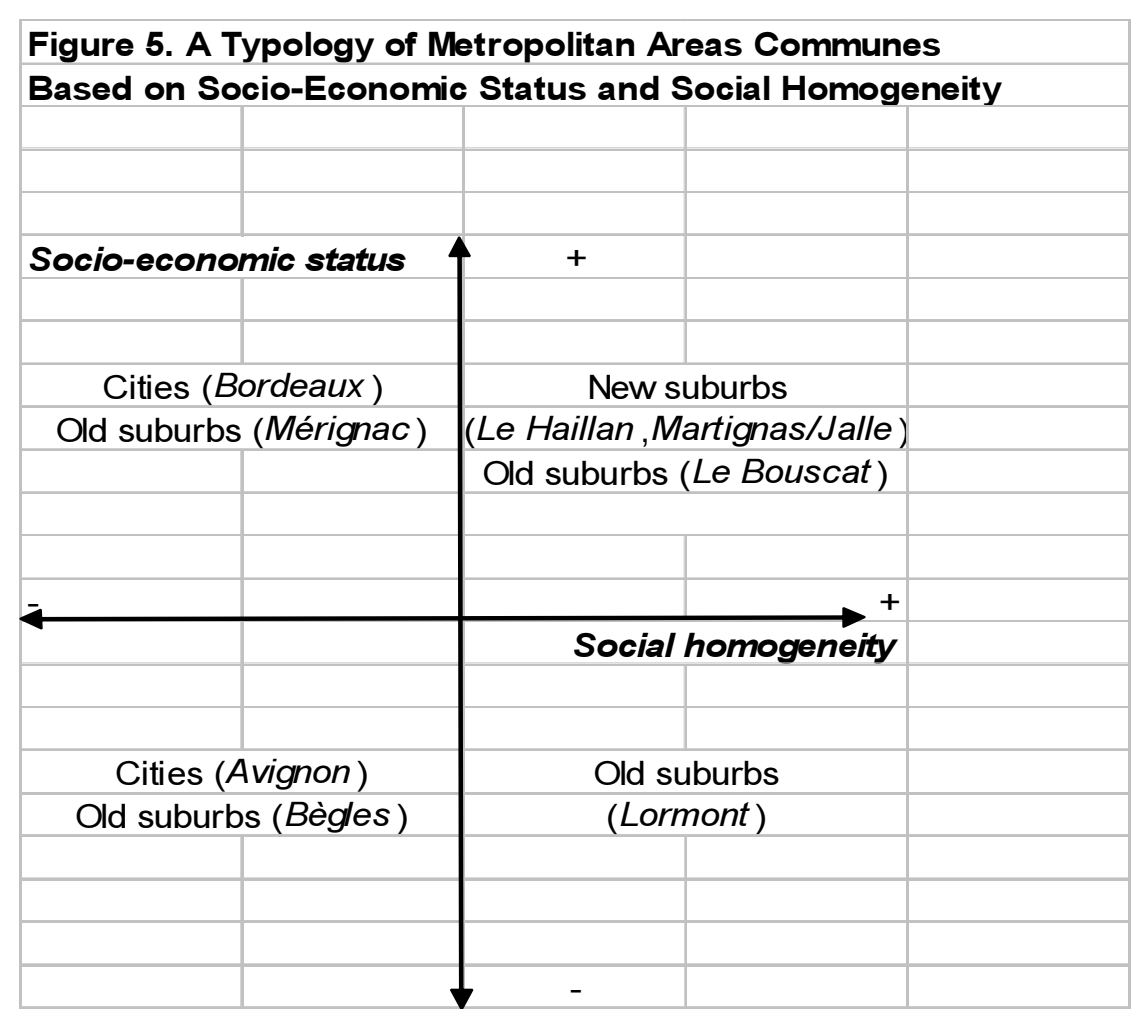

La figure 5 représente une typologie des communes des aires urbaines françaises en quatre catégories dressée selon leur niveau d'homogénéité sociale et de statut socioéconomique. Ces deux dimensions paraissent en effet fondamentales pour comprendre les logiques de ségrégation croissante à l'œuvre conditionnant les variations des comportements politiques en fonction des lieux de résidence. Les villes-centres demeurent dans l'ensemble caractérisées en France par un niveau relativement élevé d'hétérogénéité sociale : richesse et pauvreté coexistent sur leur territoire comme en témoignent, sur le territoire d'une même 
ville, la diversité des profils de leurs quartiers : il suffit de comparer le très prospère $7^{\text {ème }}$ arrondissement de Paris (revenu médian de $21.246 €$ par personne en 2000) au $19^{\text {ème }}(8.728 €)$ ou, à Marseille, le $8^{\text {ème }}(12.424 €)$ au $3^{\text {ème }}$ arrondissement $(4.028 €)$. Les old suburbs regroupent les banlieues dont le développement s'est effectué pour l'essentiel avant les années 1980. On ne peut pas les réduire à un modèle unique tant elles sont socio-économiquement différenciées. Elles se répartissent par conséquent dans les quatre catégories de communes: elles peuvent en effet être socialement hétérogènes et riches comme Mérignac dans l'aire urbaine de Bordeaux, ou relativement pauvres mais en voie de gentrification comme Bègles ; on trouve également des old suburbs socialement homogènes, composées essentiellement d'ouviers et d'employés comme Lormont, ou à dominante de population aisée comme Le Bouscat. Par contre, les new suburbs présentent un profil beaucoup plus spécifique puisque, à l'image du Haillan et encore plus de Martignas-sur-Jalle, elles sont socialement homogènes et fortement ségrégées en faveur des couches les plus qualifiées et aisées de la population.

La ségrégation socio-économique entre communes n'est donc pas un phénomène nouveau : il y a trente ans déjà, on savait très bien, dans l'aire urbaine de Bordeaux, que selon que vous habitiez Le Bouscat ou Lormont, vous tendiez à être classé comme plutôt riche ou plutôt pauvre. Mais, depuis, les dynamiques de ségrégation réisidentielle se sont à la fois diversifié, affiné, et accentué. Les new suburbs sont la plus claire manifestation de cette évolution. Deux conséquences majeures sont à attendre de cette suburbanisation ségrégative sur les comportements politiques: le déclin de la participation politique d'une part, le développement du conservatisme fiscal d'autre part.

Plus les communes sont ségrégées les unes par rapport aux autres et par conséquent socialement homogènes, moins les enjeux politiques municipaux feront l'objet de débats et de conflits faute de dissensus, conduisant à une faible mobilisation politique des citoyens. L'atténuation des clivages sociaux et de la conflictualité politique favorise nettement l'abstention aux élections locales (comme le montre l'analyse anglo-française multivariée de Hoffmann-Martinot, Rallings and Thrasher 1996). On peut donc supposer que toutes choses égales par ailleurs - c'est-à-dire à mobilité géographique et structure de la compétition politique équivalentes - la participation électorale devrait être supérieure dans les villescentres et les bannlieues socialement hétérogènes que dans les banlieues socialement homogènes. Parmi ces-dernières, on peut également formuler l'hypothèse que la surreprésentation des propriétaires dans les new suburbs - une catégorie de citoyens plus 
intéressée par les enjeux politiques locaux pour des raisons liées aux effets directs des politiques municipales (fiscales, urbanistiques, d'infrastructures,...) sur leur patrimoine immobilier - devrait favoriser un niveau relativement plus élevé de participation électorale que dans les old suburbs.

Jusque dans les années 1980 et à la mise en œuvre progressive de la réforme de décentralisation, la fiscalité locale était étroitement contrôlée par l'Etat notamment à travers la fixation des taux. Depuis cette époque, elle a sensiblement augmenté et constitue un élément désormais déterminant dans les choix de localisation des entreprises et des ménages, ce qui singularise sans doute la France par rapport aux autres pays européens. Comme le rappelle Gilbert (1999), d'une part l'autonomie fiscale locale est particulièrement élevée en France à travers la marge de manoeuvre dont disposent les collectivités locales pour fixer les quatre impôts directs (taxe professionnelle, taxe d'habitation, taxe foncière sur les propriétés bâties, et taxe foncière sur les propriétés non bâties), et d'autre part cette autonomie varie considérablement d'un territoire à l'autre en raison du degré très élevé de fragmentation géopolitique. En d'autres termes, la théorie du vote avec les pieds de Charles Tiebout, dont on connaît certes les limites d'application, trouve en France un terrain d'illustration et de manifestation certainement plus pertinent que dans des pays européens territorialement plus consolidés où l'autonomie fiscale municipale est plus limitée comme l'Allemagne ou les Pays-Bas. Le citoyen français peut espérer trouver sur « le marché de la localisation » un large éventail de rapports qualité/ prix lui permettant d'arbitrer en fonction de ses préférences entre un nombre élevé de combinaisons de coûts, d'avantages et d'aménités. Au sein d'une même région urbaine, cette offre tendra à être d'autant plus diversifiée que le territoire global sera fragmenté. A niveau équivalent de coûts (foncier et de transports) et d'aménités, les ménages relativement aisés souhaitant s'installer en banlieue privilégieront les communes à faible pression fiscale, souvent localisées à l'extérieur des agglomérations et de leurs structures intercommunales telles que les communautés urbaines ou les districts. L'attractivité dans l'aire urbaine de Bordeaux d'une banlieue comme Cestas, qui borde le territoire de la lourde et chère Communauté Urbaine de Bordeaux (CUB) sans lui appartenir (par conséquent à la fois proche géographiquement et distante fiscalement), tient en grande partie précisément à la charge fiscale locale sensiblement moins lourde qu'elle impose à ses habitants en comparaison des 27 communes de la CUB. 
Les stratégies fiscales individuelles de ce type tendent à se développer en particulier dans les aires urbaines dont la situation socio-économique de la ville-centre est défavorable ou en détérioration et la contraint à augmenter sa pression fiscale. La poursuite de la décentralisation, qui se traduit par un progressif désengagement financier de l'Etat notamment pour le financement d'équipements lourds de centralité (opéras, musées, bibliothèques,...), ainsi que la concentration croissante de populations dépendantes dans les villes-centres (personnes âgées, pauvres notamment) ont sensiblement accru le fiscal stress pesant sur les villes-centres. En comparaison, la charge fiscale locale par habitant apparaît généralement bien moindre dans des banlieues à population réduite, aisée, et homogène, bénéficiant en outre de la présence de quelques entreprises dynamiques contribuant substantiellement au budget municipal via la taxe professionnelle. L'objectif de payer moins d'impôts dans une commune menant une politique modérée de dépenses détermine par conséquent le comportement de localisation des ménages dans les new suburbs, plutôt que dans les autres communes urbaines. Même si l'on doit examiner avec une grande prudence la relation entre composition sociale et couleur politique d'une municipalité, remarquons cependant que la migration de classes moyennes et supérieures à la périphérie tend objectivement à réduire l'assise électorale de la droite. Il y a encore vingt ans, la structure plus bourgeoise des villescentres facilitait leur contrôle par des majorités de droite : c'était le cas en 1983 des deux tiers environ (27) des centres de nos 42 aires urbaines. Vingt ans pls tard, la droite n'en dirige plus que la moitié (21).

Ce conservatisme fiscal atteint des degrés et s'exprime politiquement selon des formes différentes selon le type de banlieues. Rejoignant l'argument de Gainsborough (2001) appliqué aux Etats-Unis, notre hypothèse est que les nouveaux suburbains, étant donné leur choix de résidence et d'existence, sont porteurs de préférences économiques et fiscales conservatrices influençant directement le choix de leurs leaders locaux et les policy outputs de leurs municipalités. Ce conservatisme fiscal plus ou moins explicite et accentué peut certes se combiner avec un libéralisme socio-culturel en recomposant une New Political Culture (Clark et Hoffmann-Martinot 1998) susceptible d'être incarnée par des élus indépendants et de droite, mais aussi par des leaders de gauche modérés tels que des socialistes pragmatiques. Aussi générale soit-elle, une telle évolution ne peut être ramenée ou réduite à un modèle unique et uniforme de conservatisme. Pour ces new suburban, qui ne sont pas uniquement mûs par le self-interest, il ne s'agit pas de bannir ou de limiter systématiquement tout interventionnisme public municipal: ils pourront au contraire souhaiter le développement 
d'équipements scolaires et socio-culturels. Si leur sociotropisme leur permet de rechercher non pas la simple conservation mais aussi l'amélioration de leur localité, la structure sociale homogène et l'esprit de celle-ci ne doivent cependant pas être fondamentalement modifiés. 


\section{REFERENCES}

Altshuler Alan, William Morrill, Harold Wolman, and Faith Mitchell (Eds.). 1999. Governance and Opportunity in Metropolitan America. Washington, D.C. : National Academy Press.

Andan Odile, Pascal Pochet, Jean-Louis Routhier, and Bernard Schéou. 1999. Stratégies de localisation résidentielle des ménages et mobilité domicile-travail. Rapport de recherche pour la DRAST, 1999, 203 p. + annexes.

Bessy Pascale. 1998. « Parcours résidentiels des jeunes ménages dans l'espace urbain », in : Denise Pumain and Marie-Flore Mattei (coordonné par), Données urbaines 2. Paris : Anthropos : 85-92.

Brueckner Jan K., Jacques-François Thisse et Yves Zenou. 1999. « Why is Central Paris Rich and Downtown Detroit Poor? An Amenity-Based Theory », European Economic Review, 43, 91-107.

Burgel Guy. 2003. « La ville contemporaine de la seconde guerre mondiale à nos jours », in : Pinol Jean-Luc (sous la direction de). 2003. Histoire de l'Europe urbaine. Paris : Seuil (Vol. 2) : 555-807.

Caruso Geoffrey. 2003. «Un modèle cellulaire et dynamique de dispersion et ségrégation spatiale périurbaine », communication au XXXIXème Colloque de l'Association de Science Régionale de Langue Française, Lyon, 1-3 Septembre 2003.

Cavailhès Jean and Florence Goffette-Nagot. 2003. " Parc de logements et revenus dans les aires urbaines », in : Denise Pumain and Marie-Flore Mattei (coordonné par), Données urbaines 4. Paris : Anthropos : 181-197.

Cavailhès Jean, Dominique Peeters, Evangelos Sékeris, Jacques-François Thisse. 2002. La ville périurbaine. Université de Franche-Comté, Centre d'Economie et Sociologie appliquées à l'Agriculture et aux Espaces Ruraux, Working Paper.

Chenu Alain and Nicole Tabard. 1994. «Les transformations sociales du territoire », INSEE Première, $\mathrm{n}^{\circ} 341$.

Clark Terry and Vincent Hoffmann-Martinot (Eds.). 1998. The New Political Culture. Boulder, Co. : Westview Press (Urban Policy Challenges).

Dreier Peter, John Mollenkopf and Todd Swanstrom. 2001. Place Matters. Metropolitics for the Twenty-First Century. Lawrence, Kansas : University Press of Kansas.

Dubois-Taine Geneviève (sous la direction de). 2002. La ville émergente. Résultats de recherches. Paris : Plan Urbanisme Construction Architecture

Fijalkow Yankel. 1998. «Mesurer le surpeuplement des logements à Paris », in : Denise Pumain and MarieFlore Mattei (coordonné par), Données urbaines 2. Paris : Anthropos : 93-99.

Gainsborough Juliet F. 2001. Fenced Off: The Suburbanization of American Politics. Washington, D.C. : Georgetown University Press.

Gilbert Guy. 1999. "L'autonomie financière des collectivités locales est-elle en question? », in : Caisse des Dépôts et Consignations, Quel avenir pour l'autonomie des collectivités locales? La Tour d'Aigues : Editions de l'Aube : 159-190.

Hayden Dolores. 2003. Bulding Suburbia. Green Fields and Urban Growth 1820-2000. New York : Pantheon Books.

Hintze Otto. 1962. "Staatenbildung und Kommunalverwaltung", in : Staat und Verfassung. Gesammelte Abhandlungen zur allgemeinen Verfassungsgeschichte. Göttingen : Vandenhoeck \& Ruprecht : 216-241.

Hoffmann-Martinot Vincent. 2003. "The French Republic, one yet Divisible? », in : Norbert Kersting and Angelika Vetter (Eds.), Reforming Local Government In Europe : Closing the Gap between Democracy and Efficiency? Opladen : Leske+Budrich (Urban Research International) : 157-182. 
Hoffmann-Martinot Vincent. 2002. «Décentralisation : les convergences franco-allemandes », Allemagne d'Aujourd'hui, octobre-décembre, $\mathrm{n}^{\circ} 162: 329-341$.

Hoffmann-Martinot Vincent. 2000. «The fragmented democracy of French big cities », in : Oscar W. Gabriel, Vincent Hoffmann-Martinot and Hank Savitch (Eds.). Urban Democracy. Opladen : Leske+Budrich (Städte und Regionen in Europa, 1) : 261-311.

Hoffmann-Martinot Vincent, Colin Rallings and Michael Thrasher. 1996. « Comparing local electoral turnout in Great-Britain and France : More similarities than differences? », European Journal of Political Research, 30, September $1996: 241-257$.

Julien Philippe. 2003. «L'évolition des périmètres des aires urbaines 1968-1999», in : Denise Pumain and Marie-Flore Mattei (coordonné par), Données urbaines 4. Paris : Anthropos : 11-20.

Julien Philippe. 2000. « Mesurer un univers urbain en expansion », Economie et Statistique, n³36, $6:$ 1-32.

Kübler Daniel and Andreas Ladner. 2003. «Local Government Reform in Switzerland. More for than $b y$ - but what about of? ", in : Norbert Kersting et Angelika Vetter (Eds.). Reforming Local Government in Europe : Closing the Gap between Democracy and Efficiency? Opladen : Leske und Budrich : 137-156.

Ladner Andreas and Reto Steiner. 2003. «Die Schweizer Gemeinden im Wandel », Schweizerische Zeitschrift für politische Wissenschaft, 9, (1),: 233-259.

Le Toqueux Jean-Luc. 2003. "Lieux de résidence et sentiment d'insécurité », in : Denise Pumain and MarieFlore Mattei (coordonné par), Données urbaines 4. Paris : Anthropos : 147-154.

Nathan Richard P. and Charles Adams. 1989. "Four Perspectives on Urban Hardship », Political Science Quarterly, Vol. 104, No. 3, Fall : 483-508.

Nathan Richard P. and Charles Adams. 1976. «Understanding Central City Hardship », Political Science Quarterly, Vol. 91, No. 1, Spring : 47-62.

Orfield Myron. 2002. American Metropolitics : The New Suburban Reality. Washington, D.C.: Brookings Institution Press.

Péguy Pierre-Yves. 2000. Analyse économique des configurations urbaines et de leur étalement. Thèse en Sciences Economiques. Université de Lyon 2. (http://theses.univ-lyon2.fr/Theses2000/peguy_py/these.html).

Pinol Jean-Luc (sous la direction de). 2003. Histoire de l'Europe urbaine. Paris : Seuil (2 volumes).

Putnam Robert D. 2000. Bowling Alone. The Collapse and Revival of American Community. New York : Simon $\&$ Schuster.

Rouxel Michel. 2003. « La carte de France des revenus déclarés », INSEE Première, nº00, mai.

Sauvage André, Agnès Lemoine, and Stéphane Chevrier. 2002. «Quand se modifie la puissance émerge un autre territoire », in : Geneviève Dubois-Taine (sous la direction de). La ville émergente. Résultats de recherches. Paris : Plan Urbanisme Construction Architecture : 53-61.

Sellers Jefferey. 1999. "Public Goods and the Politics of Segregation: An Analysis and Cross-National Comparison », Journal of Urban Affairs, Vol. 21, No. 2 : 237-262.

Thisse Jacques-François, Etienne Wasmer and Yves Zenou. 2003. Ségrégation urbaine, logement et marchés du travail. Dijon : Université de Franche-Comté, Centre d'Economie et Sociologie appliquées à l'Agriculture et aux Espaces Ruraux, Working Paper.

U.S. Census Bureau. 2002. 2002 Census of Governments. Vol.1, Number 1, Government Organization. GC021(1). Washington, D.C. : U.S. Census Bureau, December (http://www.census.gov/prod/2003pubs/gc021x1.pdf). 
Zeigler Don J. and Stanley D. Brunn. 1980. « Geopolitical Fragmentation and the Pattern of Growth and Need », in S. D. Brunn and J. O. Wheeler (Eds.), The American Metropolitan System: Present and Future, New York: John Wiley, Scripta Series in Geography (with Don J. Zeigler), pp. 77-92. 